\title{
Crossed beam energy transfer in the presence of laser speckle ponderomotive self-focusing and nonlinear sound waves
}

S. Hüller, ${ }^{1,2, \text { a) }}$ G. Raj, ${ }^{1,2}$ W. Rozmus, ${ }^{3,4}$ and D. Pesme ${ }^{1}$

${ }^{1)}$ Centre de Physique Théorique (CPHT), CNRS, Ecole Polytechnique, IP Paris, 91128 Palaiseau,

France

${ }^{2)}$ LOA, ENSTA ParisTech, CNRS, Ecole polytechnique, 91762 Palaiseau, France

${ }^{3)}$ Theoretical Physics Institute, Department of Physics, University of Alberta, Edmonton, Alberta,

Canada T6G $2 E 1$

4) High Energy Density Science Division, SLAC National Accelerator Laboratory, Menlo Park, California 94025,

USA

Crossed beam energy transfer, CBET, is investigated by taking into account the speckle structure of smoothed laser beams that overlap in a plasma with an inhomogeneous flow profile. Using the two-dimensional simulation code HARMONY, it is shown how ponderomotive self-focusing of laser speckles in crossing beams can significantly affect the transfer of energy from one beam to the other. The role of plasma flow on speckle self-focusing is investigated and revisited, in particular its consequences in terms of redirection and increasing angular spread of the laser beams due to beam bending and plasma-induced smoothing, respectively. At close-to-sonic flow the onset of self-focusing in the beam speckle structure occurs at considerably lower beam intensities than expected for the case without flow. CBET and speckle self-focusing can hence occur together when two crossed beams with equal frequency will resonantly exchange energy via their ponderomotively driven density perturbations flowing with sound speed. From the simulations it is found that consequences of ponderomotive self-focusing can be expected above an average intensity threshold scaling as $I_{L} \sim 2 \times 10^{14} \mathrm{Wcm}^{-2}\left(\lambda_{0} / 1 \mu \mathrm{m}\right)^{-2}\left(T_{e} / \mathrm{keV}\right)$ with impact on the spatial and temporal coherence of the transmitted light. The density perturbations due to the ponderomotive force of the crossing beams can locally be enhanced in selffocusing speckles, partly leading to shock-like structures. These structures eventually increase the effect of plasmainduced smoothing and are at the origin of the stronger angular spread.

\section{INTRODUCTION}

In the two approaches to laser-driven inertial confinement fusion (ICF) experiments, namely the direct-drive $\mathrm{e}^{1-5}$ and the indirect-drive ${ }^{6-8}$, crossed beam energy transfer (CBET) is of prime importance since it governs the coupling of laser energy to plasma. The two schemes of ICF involve multiple laser beams crossing each other at different angles and directions. For indirect drive, beams cross in the low density plasma of the laser entrance hole while propagating toward the hohlraum wall; in direct drive, they cross in the coronal plasma of the fuel capsule at considerably higher density. In both approaches and especially for the direct drive ICF, plasma flow plays an important role in defining resonance conditions and plasma response during the CBET. In addition to the context of ICF, CBET is also the principal mechanism for the amplification of a laser pulse of ps duration by a pump laser pulse $\mathrm{e}^{9,10}$ and in recent pump-probe plasma diagnostic experiments ${ }^{11}$ and theory ${ }^{12}$. In the laser pulse compression and amplification schemes, the energy transfer is devised to occur in pre-formed (mostly gas-jet) plasmas to obtain spatiotemporal growth of the probe.

The laser-plasma configurations in ICF experiments of concern for CBET involve two laser beams with wave vectors and frequencies $\left(\vec{k}_{1}, \omega_{1}\right)$ and $\left(\vec{k}_{2}, \omega_{2}\right)$, crossing at an angle $\theta$ and leading to induced ${ }^{13-15}$ or stimulated Brillouin scattering (SBS) of one beam into the other ${ }^{16-19}$. The laser light beams scatter off the grating of ion acoustic waves (IAW) produced

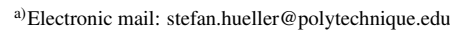

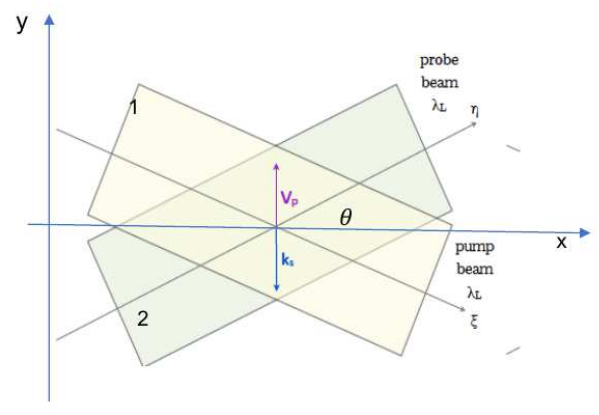

FIG. 1. Schematic sketch of the configuration of two laser beams crossing under the angle $\theta$ in a plasma with flow. The 'pump' and the 'probe' laser beam ponderomotively drive periodic density perturbations having the wave vector $\vec{k}_{s}$. For two beams with the same wave length and for plasma flow with a component $\vec{v}_{p}$ parallel to $\vec{k}_{s}$, the conditions for resonant CBET-SBS are fulfilled around the spatial domain of sonic flow, i.e. $\left|\vec{v}_{p}\right| \simeq c_{s}$. A basis of coordinates $\eta$ and $\xi$, non-orthogonal for $\theta \neq \pm 90^{\circ}$, can be defined which is used for the solutions derived in section III A.

by the ponderomotive force of the two beams. In most of the experimental configurations, plasmas are inhomogeneous and are flowing with a velocity $\vec{v}_{p}$. Therefore, the CBET requires that the three-wave SBS resonance conditions are fulfilled for wave vectors (momentum) and frequencies (energy): $\vec{k}_{s} \equiv \vec{k}_{1}-\vec{k}_{2}$ and $\omega_{1}-\omega_{2} \equiv \omega_{s}+\vec{k}_{s} \cdot \vec{v}_{p}$ respectively, where $\omega_{s}$ 
and $k_{s} \simeq 2\left|k_{1}\right| \sin (\theta / 2)$ are the IAW frequency and wave number for light beams crossing at the angle $\theta$. As the result of CBET, the power distribution in the two beams is changed; this can seriously affect the laser energy coupling to plasmas in both the indirect and direct drive ICF schemes.

Our study in this article is focused on two important aspects that prove to be important for CBET, namely

(1) the speckle (or hot spot) structure of the laser beams and (2) the role of plasma flow.

Currently all ICF experiments are carried out with 'smoothed' laser beams resulting from spatial and/or spatiotemporal smoothing techniques; in particular, in the case of spatial smoothing only, random phase plates (RPP) ${ }^{20,21}$ induce spatial incoherence in the laser beams. On a coarse scale smoothed beams show a smooth average intensity profile in their cross section, while on the fine scale of the laser wavelength $\left(\lambda_{0}\right)$ they have a speckle structure with a known statistical distribution of the speckle peak intensity $I_{\mathrm{sp}}{ }^{20-22}$. The goal of using smoothed laser beams is to control the onset of self-focusing in speckles, so as to restrict it to an energetically unimportant, small percentage of intense laser speckles.

While the theory of crossed beam power transfer between speckle beams has been developed in Refs. 23 and 24, most of the current modelings of CBET between multiple RPP beams in ICF experiments are described by averaging over the realistic beam speckle structure ${ }^{6,25,26}$. Studies on the role of speckles and their self-focusing for CBET in presence of plasma flow are relatively recent. ${ }^{4,16,27}$ In the regime of moderate laser intensity of the crossing beams, i.e. when no self-focusing in laser speckles arises, recent studies ${ }^{19,28}$ have shown that the role of speckles in the energy transfer is merely of statistical nature: the deviation in the energy transfer arising from different RPP realisations decreases with the number of speckles in the crossing volume, and the expectation value of the transfer corresponds to the value obtained when assuming the average intensity of each beam.

The onset of ponderomotive self-focusing (PSF) in speckles arises if the power in a speckle, $P$, exceeds the critical power for PSF, $P_{c}$. For direct-drive configurations ${ }^{2-4}$ beams essentially cross in counterpropagating geometry so that energy exchange can be computed on the basis of standard 1D models for backward SBS ${ }^{29,30}$. PSF in such configurations appears in the individual beams and on a longer time scale than CBET.

The scenario is different for indirect drive ICF where PSF and CBET occur on a similar time scale. Furthermore, the zone of sonic flow, in the vicinity of which efficient CBET takes place between the crossing beams, is relatively large. It is known that the transverse plasma flow reduces the threshold for PSF in the sub-sonic regime. ${ }^{31-33}$ In the vicinity of the spatial domain where the plasma flow is sonic, the so-called effect of beam-bending occurs ${ }^{23,33-35}$ where a beam is redirected into a direction different from its incident direction. The latter has consequences to intense speckles in smoothed laser beams: speckles located in the region of overlapping beams and close to the region of sonic flow will eventually be redirected towards the direction of the other beam.

On the other hand beam bending slows down the plasma flow by momentum conservation and can locally lead to den- sity profile steepening. Density and velocity perturbations in the plasma that are enhanced by the PSF are carried by the plasma flow away from the localized ponderomotive force of the crossing beams and can eventually develop shock-like structures. ${ }^{36-38}$ These structures will scatter electromagnetic waves, enhance levels of ion acoustic fluctuations over wide range in the wave-vector spectrum, and contribute to enhanced plasma-induced smoothing of the transmitted light. In the transmitted light beams the latter leads to broadening in the angular aperture and introduces (or increases) temporal incoherence, resulting in non-negligible temporal bandwidth.

Two smoothed beams crossing in an expanding inhomogeneous plasma, as sketched in Fig. 1, are relevant to indirectdrive ICF experiments, where the plasma at the laser entrance hole is weakly inhomogeneous, both in density and flow ${ }^{39-44}$. Experimental studies with similar configurations have been undertaken recently ${ }^{45,46}$, but also at smaller angles ${ }^{47,48}$, or partially at larger angles 49 .

In this article we show how important becomes ponderomotive self-focusing (PSF) in laser speckles when plasma flow is present. For the case of two crossing RPP beams we show that in inhomogeneous plasmas ${ }^{16}$ speckle self-focusing and the deflection of speckles lead to a significant effect on the CBET which increases with the laser beam intensity. We have not considered spatio-temporal smoothing as 'smoothing by spectral disperion' (SSD) in the current study, but we discuss the impact of temporal incoherence on our results.

The article is organized as follows: the model used for describing CBET for beams with speckle structure in a plasma, with the details concerning the fundamental equations used in our modelling with the code HARMONY is presented in section II. Particular attention is given to the ponderomotive coupling in section III. We also recall the essential theory for beam-to-beam CBET in III A. In III B we will develop a model to explain ponderomotively induced density perturbations that are seen in speckle beams. The simulation results for several laser plasma parameters as obtained from the code HARMONY are presented in section IV. In this section we also discuss the role played by laser speckle structure, ponderomotive self-focusing, and density shock structure on CBET. Conclusions are presented in section $\mathrm{V}$.

\section{MODELING CBET BETWEEN TWO BEAMS IN A FLOWING PLASMA}

We will describe in the following the interaction between two laser beams crossing at the angle $\theta$ in an inhomogeneous plasma. Figure 1 shows the particular configuration that corresponds to two ' $s$ '-polarized beams crossing at a relatively small angle $\theta$, and having their common wave vector component along the positive $x$ direction, while the ponderomotively generated ion acoustic waves (IAWs) propagate along the $y$ direction. Such a configuration of crossing beams may be relevant to the basic element of the geometry of many crossing beams at laser entrance holes ('LEH') in the indirect drive ICF experiments. We have also chosen an inhomogeneous plasma flow profile, $v_{p, y}(y) \vec{e}_{y}$, with the dominating direction of flow direction along the $y$-axis. This is 
one of several possible situations that may be encountered in LEHs flow profiles ${ }^{50}$, and it is the one with the strongest possible exchange between beams that cross at a small angle. For two 's'-polarized beams, with wave vectors and frequencies $\left(\vec{k}_{1}, \omega_{1}\right)$ and $\left(\vec{k}_{2}, \omega_{2}\right)$, the SBS matching conditions are satisfied when $v_{p, y}(y) / c_{s}=\left(\omega_{1}-\omega_{2}-\sigma \omega_{s}\right) /\left(c_{s} k_{s}\right)$, where $\sigma$ is the sign of $\omega_{1}-\omega_{2}-\left(\vec{k}_{1}-\vec{k}_{2}\right) \cdot \vec{v}_{p}$, and where $c_{s} \equiv\left[\left(c_{s e}^{2} /\left(1+k_{s}^{2} \lambda_{D e}^{2}\right)+3 v_{i}^{2}\right]^{1 / 2}\right.$ is the IAW velocity, with $c_{s e} \equiv\left(Z T_{e} / m_{i}\right)^{1 / 2}$, where $T_{e}$ is the electron temperature, $\lambda_{D e}$ the Debye length, $v_{i}$ the ion thermal velocity, $m_{i}$ and $Z$ are the ion mass and charge number, respectively.

\section{A. Model equations}

In the following we express the complex electric field envelope as the superposition of two beams incident at the angles $\pm \theta / 2$ to the $\mathrm{x}$-axis, where $\vec{k}_{j \|}$ and $\vec{k}_{j, y}$ are the parallel and transverse components of the wave vectors, respectively, with $\left|\vec{k}_{j \|}\right|=\left|\vec{k}_{j}\right| \cos (\theta / 2),\left|\vec{k}_{j, y}\right|=\left|\vec{k}_{j}\right| \sin (\theta / 2)$ and $\left|\vec{k}_{j}\right|=\left(\omega_{j}^{2}-\omega_{p}^{2}\right)^{1 / 2} / c(j=1,2)$. The electric field can then be written as

$$
E(\vec{x}, t)=\frac{\hat{E}}{2} e^{i \vec{k}_{1 \|} \cdot \vec{x}}\left[a_{01} e^{-i \omega_{1} t+i \vec{k}_{1, y} \cdot \vec{y}}+a_{02} e^{-i \omega_{2} t+i \vec{k}_{2, y} \cdot \vec{y}}\right]+c c,
$$

where $a_{01}$, and $a_{02}$ are the electric field amplitudes of both beams normalized to the field strength $\hat{E}$. For not too large angles $\theta$ between the two beams, the paraxial approximation can be used, and for $\left|\omega_{1}-\omega_{2}\right| \equiv 0$ and $\vec{k}_{2, y}=-\vec{k}_{1, y}$ the propagation of the incoming beams can be described by paraxial equations for $a(\vec{x}, t) \equiv a_{01} \exp \left\{\overrightarrow{i k}_{1, y} \cdot \vec{y}\right\}+a_{02} \exp \left\{-i \vec{k}_{1, y} \cdot \vec{y}\right\}$, or, alternatively, individually for $a_{01}, a_{02}$, coupled to the plasma density perturbations ${ }^{51}$

$$
\left[2 i \omega_{1}\left(\partial_{t}+v_{g x} \partial_{x}\right)+c^{2}\left(\nabla^{2}\right)_{\perp}\right] a(\vec{x}, t)=\omega_{p}^{2} \frac{\delta n}{n_{e}} a(\vec{x}, t)
$$

where $v_{g x} \equiv v_{g} \cos (\theta / 2), \omega_{p}=\left(n_{e} e^{2} / \varepsilon_{0} m_{e}\right)^{1 / 2}$ is the electron plasma frequency; $n_{c}=\varepsilon_{0} m_{e} \omega_{0}^{2} / e^{2}$ denotes the critical density, $m_{e}$ and $e$ being the plasma electron mass and charge respectively, $\delta n=\left(n-n_{e}\right)$ is the density perturbation about the equilibrium density $n_{e}$. Note, that Eq. (2) describes the evolution of the electromagnetic wave amplitudes in the paraxial approximation on the scale of hydrodynamical evolution and long wavelength IAW response of $\delta n / n_{e}$. The high-frequency response for IAWs due to backscattered SBS is treated in HARMONY via a harmonic decomposition ${ }^{52}$, that one has to consider for large angles $\theta \sim 180^{\circ}$. In HARMONY we solve Eq. (2) by imposing a boundary condition for the entering laser light at $x=0$ for $a(x=0, y, t)$.

The plasma dynamics is described by the standard hydrodynamic equations in the isothermal approximation,

$$
\begin{aligned}
& \partial_{t} n+\nabla \cdot(n \vec{v})=0, \\
& {\left[\partial_{t}+\vec{v} \cdot \nabla\right] \vec{v}+c_{s}^{2} \frac{\nabla n}{n}+2 v_{s} \vec{v}=-c_{s e}^{2} \frac{\nabla U}{T_{e}},}
\end{aligned}
$$

where $U$ stands for the ponderomotive potential and $v_{s}$ for the ion acoustic damping. In our simulations we assume for $v_{s}$ a linear wave-number dependence, and $n v_{s} \vec{v}$ is computed in Fourier space with $v_{s}\left(k_{s}\right)=\hat{v} \omega_{s}\left(k_{s}\right)$, accounting for Landau damping 51,52 .

For the electric field resulting from the superposition of two beams of equal frequency, $a(\vec{x}, t) \equiv a_{01} \exp \left\{\vec{k}_{1, y} \cdot \vec{y}\right\}+$ $a_{02} \exp \left\{-i \vec{k}_{1, y} \cdot \vec{y}\right\}$, the ponderomotive force $\nabla U \propto \nabla|a(\vec{x}, t)|^{2}$ can be subdivided into two separate contributions, provided that the central wave vector component in $y$ for each beam, $\left|\vec{k}_{1, y}\right|$ and $\left|\vec{k}_{2, y}\right|$ exceeds the wave number spread $\triangle k$ related to the angular aperture of each RPP beam; the latter is a function of the focusing $f$-number, namely ${ }^{21} \triangle k \equiv\left|\vec{k}_{1}\right| /\left[1+4 f^{2}\right]^{1 / 2} \sim$ $\left|k_{1}\right| /(2 f)$.

Keeping in mind the condition, that for two separate fields the wave number separation needs to be greater than the angular aperture of the fields, $\left|\vec{k}_{2, y}-\vec{k}_{1, y}\right|>\Delta k$, the ponderomotive force in the right-hand side of the equation of motion can be expressed in two distinct terms namely $\nabla U=T_{e} \Gamma \nabla|a(\vec{x}, t)|^{2} \equiv$ $\nabla U_{\text {cross }}+\nabla U_{\text {self }}$, given by

$$
\begin{aligned}
& \nabla U_{\text {cross }} / T_{e}=\Gamma \nabla\left(a_{01} a_{02}^{*} e^{2 i\left|\vec{k}_{1}\right| y \sin (\theta / 2)}+c c .\right), \\
& \nabla U_{\text {self }} / T_{e}=\Gamma \nabla\left(\left|a_{01}\right|^{2}+\left|a_{02}\right|^{2}\right),
\end{aligned}
$$

where $\Gamma=\left\langle v_{\text {osc }}^{2}\right\rangle / v_{\text {th }}^{2}=v_{\text {osc }}^{2} /\left(2 v_{\text {th }}^{2}\right)$ is the coupling coefficient which involves the thermal velocity $v_{\mathrm{th}}=\left(T_{e} / m_{e}\right)^{1 / 2}$ and the electron quiver velocity $v_{\mathrm{osc}}=e \hat{E} /\left(m_{e} \omega\right)$ of the field $\hat{E}$ to which $a_{01}$ and $a_{02}$ are normalized. In practical units the coefficient is given by $\Gamma=0.09 I_{0} \lambda_{0}^{2}\left(10^{15} \mathrm{~W} \mu \mathrm{m}^{2} / \mathrm{cm}^{2}\right) / T_{e}(\mathrm{keV})$ with $I_{0}$ standing for the beam average intensity.

The ponderomotive force contribution $\nabla U_{\text {cross }}$ acts essentially on the plasma fluid due to the beating between the two waves $a_{01}$ and $a_{02}$ in SBS. This term alone cannot account for self-interaction occurring in an individual beam. The ponderomotive force contribution denoted by $\nabla U_{\text {self }}$ is therefore the one accounting for ponderomotive self-interaction in the paraxial approximation for each individual beam. This self-interaction can be associated with self-focusing and with forward-SBS inside each beam. Note, that in contrast to the case of large angles, both contributions to the ponderomotive force have major components along the $y$-direction, i.e. across the main common propagation axis $x$.

In most of the studies on crossed-beam coupling, only the $\nabla U_{\text {cross }}$ term was considered in the description of CBET. This term is responsible for the coupling between the average beams, which we will denote as 'beam-to-beam CBET' later on in section III A. The term $\nabla U_{\text {self }}$ was mostly neglected in the context of CBET because self-focusing effects are expected to occur for laser intensities that are above those considered in laser fusion configurations. This argument has to be revisited in plasmas with the transversal flow, as it is done further on in Section III B. We will draw attention to important work that has been done in the past by considering plasmas with flow even in the presence of a single laser beam. ${ }^{31-33}$ 

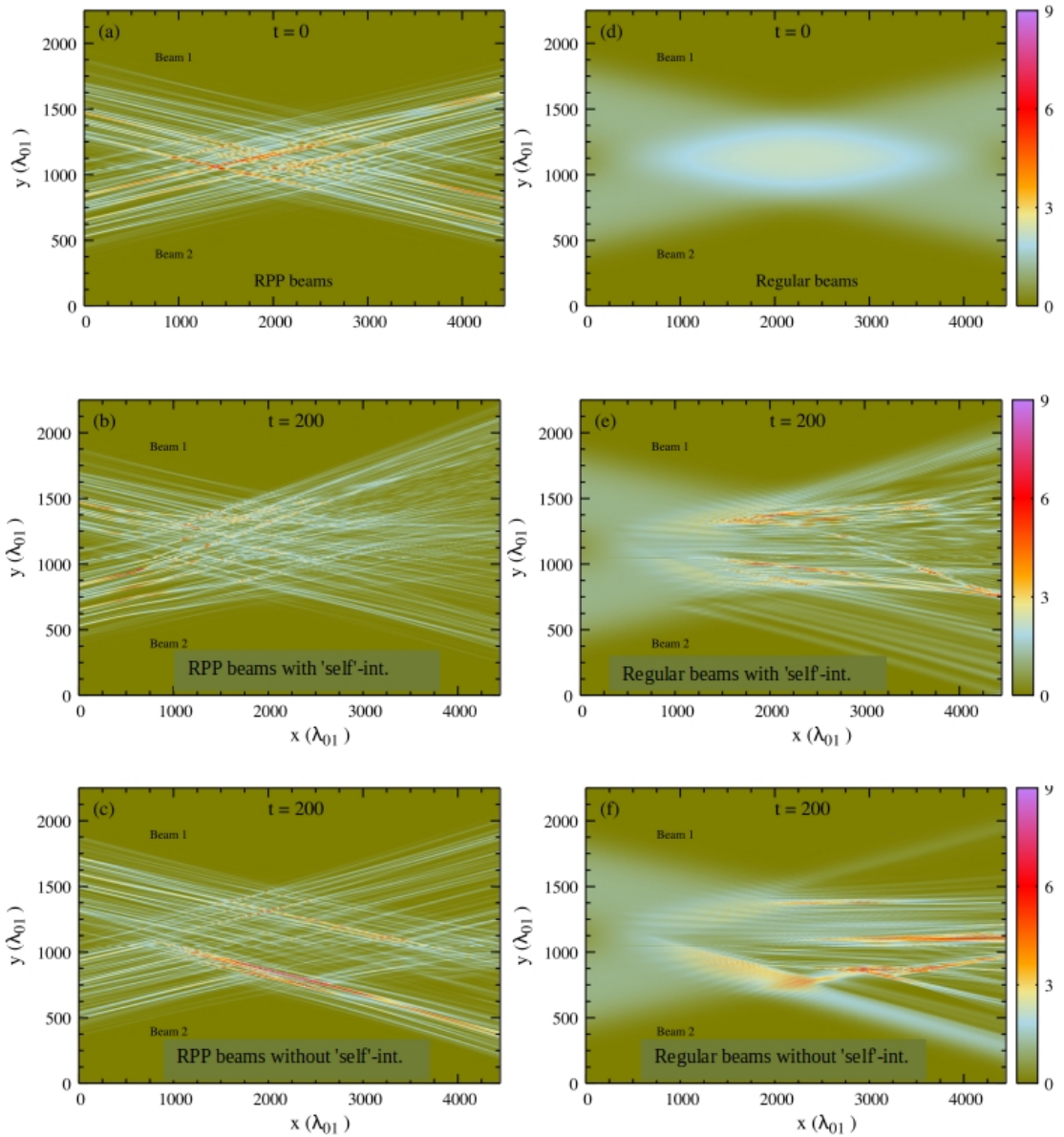

FIG. 2. Snapshots from the 2D simulations showing the superposition of intensities of the two crossed laser beams in space. In the left panels (a)-(c) are shown the results from two RPP beams, at the time instants (a) $t=0$, (b)-(c) $t=200\left(2 k_{1} c_{s}\right)^{-1}$ (corresponding to $\sim 11 \mathrm{ps}$ for $\lambda_{0}=0.35 \mu \mathrm{m}$ and $T_{e}=3 \mathrm{keV}$ ); in (b) both crossed-beam coupling and beam self-interaction ('self') are taken into account, in (c) only crossed-beam coupling. The corresponding right panels (d)-(f) show two crossed regular beams (i.e. without speckle stucture) under the same conditions as in panels (a)-(c). The color bar shows the laser beam intensities normalized to the the average value, i.e. $I_{01}=I_{02}=6 I_{0}$ with $I_{0} \lambda_{0}^{2} \equiv 10^{14} \mathrm{~W} \mu \mathrm{m}^{2} / \mathrm{cm}^{2}$.

\section{B. The simulation configuration in HARMONY}

In the two dimensional (2D) simulations with our code HARMONY ${ }^{51,52}$, we have chosen a crossing angle of $\theta=20^{\circ}$. The plasma flow profile follows a linear ramp in $y$-direction, as defined by $v_{p, y}(y) / c_{s}=\left(y-L_{y} / 2+L_{v}\right) / L_{v}$ with $L_{v}\left(=200 \lambda_{0}\right.$ in the simulations) as the gradient length ${ }^{19}$, so that, assuming equal frequencies for both beams, $\omega_{1}=\omega_{2}=\omega_{0}$, SBS matching occurs at $y=L_{y} / 2$ in the center. We have performed simulations for the case when both entering beams have the same average intensity, $I_{02}=I_{01}$. For this case, $I_{02} / I_{0}=I_{01} / I_{0} \equiv 1$, the reference intensity, $I_{0}$, corresponds for $\lambda_{0}=0.35 \mu \mathrm{m}$ light to an average laser intensity of $I_{0}=I_{L} \simeq 0.9 \times 10^{15} \mathrm{~W} / \mathrm{cm}^{2}$ at $T_{e}=3 \mathrm{keV}$. 


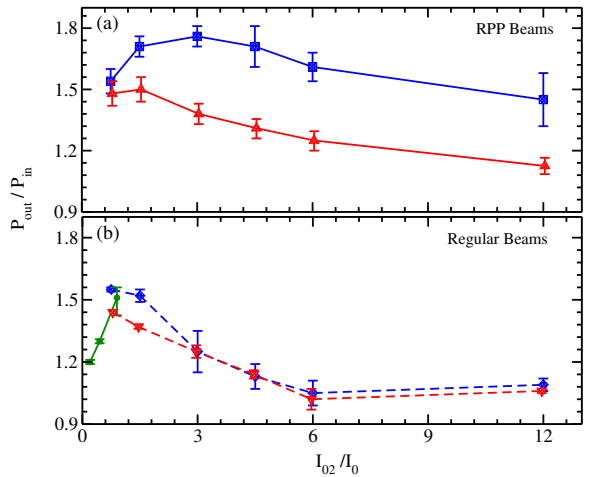

FIG. 3. Power transfer ratio for beam 2, as a function of its input flux $I_{02}$ after exchange with laser beam 1 due to CBET. Four cases are shown, (a) RPP-smoothed beams (solid lines) and (b) regular beams (dashed) with and without accounting for self-focusing (blue and red color, respectively). Both beams have initially the same flux, $I_{02}=$ $I_{01}$. The green curve corresponds to results from Ref. 19 and follows the theory ${ }^{53}$ exposed in section III A.

The laser wave amplitude, $a(\vec{x}, t)$ in Eq.(2), at the laser entrance boundary is generated via a Fourier series for two separate wave-fields for $a_{01}$ and $a_{02}$. In Fourier space, each of the wave-field contributions is centered around the wave vector components $\left|\vec{k}_{1, y}\right|=-\left|\vec{k}_{1}\right| \sin (\theta / 2)$ and $\left|\vec{k}_{2, y}\right|=\left|\vec{k}_{1}\right| \sin (\theta / 2)$, respectively. For a RPP with $i=1 \ldots N_{\mathrm{RPP}}$ elements in each component $j=1,2$, with random phases $\phi_{j, i}$, this reads $a_{0 j}(y)=e^{i \vec{k}_{j, y} \cdot \vec{y}} \sum_{k_{i}=-\triangle k}^{\triangle k}\left|\hat{a}_{j, i}\right| e^{i k_{i} y+i \phi_{j, i}}$ where the $i$-th element has the wave number $k_{i}$ and amplitude $\hat{a}_{j, i}$, with the spacing between them, $k_{i+1}-k_{i}=2 \triangle k / N_{\mathrm{RPP}}$. The width in $k_{y}$, is given by $^{21} \triangle k \equiv\left|\vec{k}_{1}\right| /\left[1+4 f^{2}\right]^{1 / 2} \sim k_{1} / 2 f$. The total field with the $a_{01}$ and $a_{02}$ components has zero elements in the Fourier series for $\left|k_{y}\right|<\left|\vec{k}_{j, y}\right|-\Delta k$, which corresponds to the angular interval around $\theta=0^{\circ}$. For an angular separation of $a_{01}$ and $a_{02}$ the condition $\triangle k<\left|\vec{k}_{1, y}\right|$ has to be fulfilled.

Note that we also have performed simulations in which a single field array was used in stead of separating the fields into two arrays. In this configuration the ponderomotive force is given simply by $\nabla U=T_{e} \Gamma \nabla|a(\vec{x}, t)|^{2}$. We have verified that the simulations of both types of configurations show the same results when both terms $\nabla U_{\text {cross }}$ and $\nabla U_{\text {self }}$ and taken into account in the configuration with two field arrays.

\section{LASER PLASMA COUPLING IN THE PRESENCE OF FLOW AND OF TWO CROSSING LASER BEAMS.}

The standard criterion for the ponderomotive self-focusing of laser speckles in RPP beams, derived in previous work (see e. g. Ref. [54]) reads

$$
\hat{P}=P / P_{c}=0.04 \eta \frac{I_{\mathrm{sp}} \lambda_{0}^{2}}{T_{e}} f^{2} \frac{n_{e}}{n_{c}},
$$

with $I_{\mathrm{sp}}$ being the peak intensity of a laser speckle given in the units of $10^{14} \mathrm{~W} / \mathrm{cm}^{2}, \lambda_{0}$ in $\mu \mathrm{m}, T_{e}$ in $\mathrm{keV}$, and $f$ as the speckle f-number; $\eta$ is a numerical factor of the order of unity, being $\eta=1.23$ in $2 \mathrm{D}$ geometry ${ }^{54}$.

However, it is important to note that this criterion cannot be applied to flowing plasmas, when CBET between laser beams of equal frequency occurs at close-to-sonic flow.

We will continue to use $\hat{P}$ in the text to indicate beam power in a speckle, even if the ponderomotive self-focusing instability may occur with flow already for values $\hat{P}<1$.

For crossing beams with speckle structure, like RPP beams, the consequences of the onset of PSF in presence of flow are manyfold: speckles located in the vicinity of sonic flow will self-focus and grow in amplitude at an angle different from their incidence angle ${ }^{31}$, a phenomenon denoted as beam bending. ${ }^{23,33-35}$ The latter may eventually lead to the deviation of the speckle into the direction of the other beam, thus corresponding to beam bending CBET; also, the density perturbations ponderomotively induced in the plasma by the numerous speckles, may develop shock-like structures characterized by steep wave fronts in presence of flow, as shown later on.

These effects become more and more pronounced with increasing laser intensity. At beam intensities slightly above the moderate intensity values considered in our previous work in Ref. ${ }^{19,28}$ the difference in the description of CBET with and without speckle structure becomes already striking.

To illustrate this we have carried out simulations with two types of beams, namely optically smoothed beams with speckle structure, and beams with a flat profile without speckles; both types of beams having the same average intensity. For the current study we use RPP beams as prototype for speckle beams.

We have not considered beams with temporal incoherence. They usually are specific to the laser facility and in most cases introduce temporal variations that, as we will demonstrate below, involve longer time scales than plasma induced correlations times of crossing RPP beams. Here, we concentrate on the essential effects of PSF in speckles on CBET. The beams without speckle structure we call 'regular beams'. The properties of such 'regular' beams are reminiscent of plane waves, i.e. with constant, steady wave fronts, with a unique phase constant, and without any randomness. Such regular laser beams are used in most of the theoretical models describing CBET on large scale laser facilities ${ }^{6,55}$. We use those beams for the comparison with RPP beam, in order to illustrate effects with and without speckle structure.

Figure 2 illustrates the difference between the two types of beams for crossing beams of equal intensities. Shown are snapshots of intensity profiles in $x$ and $y$ in the interaction region, for the case of RPP smoothed beams (with speckles, left column, (a)-(c)) and "regular" beams without speckle structure (right column (d)-(f), see for more details in the section IV on simulation results).

In the following three subsections we discuss the basic processes that come into play and that are seen in our simulations for the case of single speckle dynamics and for SBS modeling. We describe in III A the spatio-temporal evolution of the 
forward SBS responsible for CBET, in III B the modifications of the filamentation threshold due to transverse plasma flow, and in III C the linear plasma response to the ponderomotive force of PSF in the flowing plasmas leading to beam bending and steepening of the density perturbations. Although most of these results have been discussed separately before, we found this summary useful as it will inform our interpretation of the RPP crossed-beam coupling and energy transfer in large scale HARMONY simulations.

\section{A. Beam-to-beam CBET}

We will recall here results of CBET theory describing two crossing structure-less beams and refer to Ref.19 or earlier work ${ }^{53}$ for the details of the derivations. The equations describing the evolution of the laser field amplitudes $a_{01}$ and $a_{02}$ and propagating by the angle $\theta / 2$ with respect to their common axis $x$, take the following form:

$$
\begin{aligned}
& \left(\partial_{t}+2 v_{1}+\vec{v}_{g 1} . \nabla\right)\left|a_{01}\right|^{2}=+\Gamma \frac{\omega_{p e}^{2}}{\omega_{1}} \omega_{s}^{2} \mathfrak{I}\left(\frac{1}{\mathscr{D}_{s}}\right)\left|a_{01}\right|^{2}\left|a_{02}\right|^{2}, \\
& \left(\partial_{t}+2 v_{2}+\vec{v}_{g 2} . \nabla\right)\left|a_{02}\right|^{2}=-\Gamma \frac{\omega_{p e}^{2}}{\omega_{2}} \omega_{s}^{2} \mathfrak{I}\left(\frac{1}{\mathscr{D}_{s}}\right)\left|a_{01}\right|^{2}\left|a_{02}\right|^{2},
\end{aligned}
$$

$v_{1,2}$ stand for collisional damping (they can be neglected for the ICF plasma conditions), $\mathfrak{I}\left(1 / \mathscr{D}_{s}\right)$ denotes the imaginary part of the resonance denominator $1 / \mathscr{D}_{s}$ where $\mathscr{D}_{s}=$ $\left(\omega^{\prime}\right)^{2}+2 i \omega_{s}^{\prime} v_{s}-\omega_{s}^{2}$ with $\omega_{s}^{\prime}=\omega_{1}-\omega_{2}-\vec{k}_{s} \cdot \vec{v}_{p}$ describes resonant coupling between the crossing laser waves and the ion acoustic wave, $\omega_{s}^{2} \equiv k_{s}^{2} c_{s}^{2}$, and $v_{s}$ is the acoustic damping.

After a transient period of non-stationary energy exchange between the beams, Eqs. (6) can be reduced to a set of stationary equations for the beam intensities $\left|a_{01}\right|^{2}$ and $\left|a_{02}\right|^{2}$. An efficient way of expressing this coupling is realized by introducing oblique, non-orthogonal coordinates $\eta$ and $\xi$, $x=\eta \vec{e}_{x} \cdot \vec{e}_{\eta}+\xi \vec{e}_{x} \cdot \vec{e}_{\xi}$ and $y=\eta \vec{e}_{y} \cdot \vec{e}_{\eta}+\xi \vec{e}_{y} \cdot \vec{e}_{\xi}$ with $\vec{e}_{y} \cdot \vec{e}_{\eta}=$ $-\sin (\phi-\theta / 2)$ and $\vec{e}_{x} \cdot \vec{e}_{\xi}=-\sin (\phi+\theta / 2)$. Choosing $\phi=0$ as the angle between $\vec{v}_{p}$ and $\vec{k}_{s}=\vec{k}_{1}-\vec{k}_{2}$ leads to $\vec{e}_{y} \cdot \vec{e}_{\eta}=$ $\sin (\theta / 2)$ and $\vec{e}_{x} \cdot \vec{e}_{\xi}=-\sin (\theta / 2)$ in the two-dimensional geometry (see Fig. 1) of HARMONY simulations (cf. Fig. 2) For arbitrary ratios between $\left|a_{01}\right|^{2}$ and $\left|a_{02}\right|^{2}$, for which one cannot neglect depletion of either beam 1 or 2 , the set of equations to solve reads,

$$
\begin{aligned}
& \partial_{\eta} \partial_{\xi}\left|a_{01}\right|^{2}=-2 \partial_{\eta}\left(\beta(\xi, \eta)\left|a_{01}\right|^{2}\left|a_{02}\right|^{2}\right), \\
& \partial_{\xi} \partial_{\eta}\left|a_{02}\right|^{2}=2 \partial_{\xi}\left(\beta(\xi, \eta)\left|a_{01}\right|^{2}\left|a_{02}\right|^{2}\right),
\end{aligned}
$$

that simplifies to first-order differential equations when the depletion of one of the beams can be neglected, as for $\left|a_{01}\right|^{2} /\left|a_{02}\right|^{2} \gg 1$ (or, $\ll 1$ ). The function $\beta(\xi, \eta)$ accounts for the geometry of the rhombus-like shape of the crossing zone,

$$
\beta(\xi, \eta)=\frac{\gamma_{0}^{2} /\left(v_{s} v_{g, 2}\right)}{1+\frac{k_{s}^{2} c_{s}^{2}}{v_{s}^{2}}\left[Q_{0}-Q(\xi, \eta)\right]^{2}} .
$$

It depends on the spatial growth rate $\gamma_{0}^{2} /\left(v_{s} c_{s}\right)$ for SBS $\left(v_{g, 2} \equiv c_{s}\right)$ where $\gamma_{0}$ is the temporal growth rate, $\gamma_{0}^{2} \equiv$ $\left(n_{e} / n_{c}\right)\left(\omega_{1} / \omega_{s}\right) k_{s}^{2} c_{s}^{2} v_{\mathrm{osc}}^{2} /\left(4 v_{\mathrm{th}}^{2}\right)=\left(n_{e} / n_{c}\right)\left(\omega_{1} / \omega_{s}\right) k_{s}^{2} c_{s}^{2} \Gamma / 2$ with the coupling coefficient $\Gamma$ from Eqs. (4a-4b) involving the quiver velocity $v_{\mathrm{osc}}$ corresponding to the laser pump field to which $a_{01}$ and $a_{02}$ are normalized. The auxiliary functions,

$$
\begin{aligned}
Q_{0} & =\frac{\omega_{1}-\omega_{2}}{k_{s} c_{s}}-1 \\
Q(\xi, \eta) & =\left|\frac{v_{p} L_{y} / 2}{c_{s}}-\frac{\eta \sin [\phi-\theta / 2]+\xi \sin [\phi+\theta / 2]}{L_{v}}\right| \cos \phi
\end{aligned}
$$

result, with $v_{p, y}(y) / c_{s}=\left(y-L_{y} / 2+L_{v}\right) / L_{v}$, in

$$
Q(\xi, \eta)=\left|\frac{v_{p}\left(L_{y} / 2\right)}{c_{s}}+\frac{2\left(y-L_{y} / 2\right) \sin (\theta / 2)}{L_{v}}\right| .
$$

The integration over the domain of interest, namely the beam width $D$, see ${ }^{19}$ and Fig. 1, yields a spatial amplification described by the gain $G$ coefficient given by

$$
G \equiv \frac{2 \gamma_{0}^{2} \min \left\{D /(2 \sin \theta), L_{\mathrm{inh}}\right\}}{v_{s} c_{s}},
$$

where $L_{\text {inh }}=\pi\left(L_{v} v_{s} / \omega_{s}\right) /|\cos \phi \sin [\phi-\theta / 2]|$ denotes the inhomogeneity length, being $L_{\text {inh }}=\pi\left(L_{v} v_{s} / \omega_{s}\right) / \sin (\theta / 2)$ in our case. For the case $D /(2 \sin \theta)>L_{\text {inh }}$, the resulting gain is equivalent to the 'Rosenbluth' gain coefficient ${ }^{56}$.

\section{B. The role of plasma flow and of speckles for CBET}

For the case of non-flowing plasmas laser speckles are expected to self-focus with speckle power following the criterion Eq. (5). In terms of the speckle peak intensity, $I_{s p}$, it reads in practical units $\left(I_{\mathrm{sp}} / I_{0}\right) \sim 3.1\left(n_{c} / 10 n_{e}\right)(f / 8)^{-2} T_{e}(\mathrm{keV})$. It yields that speckles self-focus when $I_{\mathrm{sp}}>(f / 8)^{-2} 9 \times 10^{15} \mathrm{~W} / \mathrm{cm}^{2}$ at $\lambda_{0}=0.351 \mu \mathrm{m}$ for $n_{e} / n_{c}=0.1, T_{e}=3 \mathrm{keV}$, and $I_{0}=10^{15} \mathrm{~W} / \mathrm{cm}^{2}$. For typical ICF conditions only very few speckles would hence undergo PSF.

As already mentioned, this criterion cannot be applied in presence of plasma flow. The criterion for the onset of the filamentation instability has to be derived from the set of Eqs. (2),(3a), (3b), and Eq. (4b), taking into account the advective terms $\overrightarrow{v_{p}} \cdot \nabla \vec{v}$ and $\nabla\left(n \vec{v}_{p}\right)$ due to transverse plasma flow $\vec{v}_{y}$. Assuming for unstable modes the dependence $\propto \exp \left(q x+i k_{y} y\right)$ with respect to the $x$ and $y$ axis, and with flow along the $y$ direction, one obtains a criterion for the filamentation instability in terms of the spatial growth rate $q$.

The resulting relation reads ${ }^{31}$ (for $|q|^{2} \ll k_{0}^{2}$ )

$$
q^{2}=\frac{k_{y}^{2}}{4}\left(\frac{\left\langle v_{\mathrm{osc}}^{2}\right\rangle}{v_{\mathrm{th}}^{2}} \frac{n_{e}}{\varepsilon n_{c}} \frac{1}{1-M^{2}+i \hat{v} M}-\frac{k_{y}^{2}}{k_{0}^{2}}\right),
$$

where $M=v_{p, y} / c_{s}$ is the Mach number of the transversal flow, the damping coefficient $\hat{v}=v_{s}(k) / \omega_{s}(k)$, and $\varepsilon=1-n_{e} / n_{c}$ is the dielectric constant in the plasma. Note that when considering Gaussian beams or RPP speckles, $k_{y}$ should be larger than the minimum $k_{y, \min }=\Delta k \simeq k_{0} / 2 f$ related to their focal width. The resulting threshold for the instability, $\Re q>0$ depends on 
transverse flow via a resonance denominator, in contrast to the standard criterion without flow, i.e. $M=v_{p, y} / c_{s} \equiv 0$. For small damping, $M \hat{v} \ll\left|1-M^{2}\right|$, Eq. (12) predicts growth of the filamentation instability at wave vectors, $k_{y}$, and $\left\langle v_{\text {osc }}^{2}\right\rangle$ satisfying threshold condition, $\left(\left\langle v_{\text {osc }}^{2}\right\rangle / v_{\mathrm{th}}^{2}\right)\left(n_{e} / \varepsilon n_{c}\right)\left(k_{0}^{2} / k_{y}^{2}\right) \equiv 1-M^{2}$ for subsonic flow, $M^{2}<1$. This clearly indicates that the onset of PSF is altered with flow. For the particular case without flow, $M=0$, this criterion is equivalent to the onset of PSF, Eq. (5), for speckles, by associating $P_{c} \sim v_{\mathrm{th}}^{2} n_{c} / n$ with the critical power and $P_{\mathrm{sp}} \sim v_{\mathrm{osc}}^{2}\left(k_{0}^{2} / k_{y}^{2}\right)$ with the speckle power assuming $k_{0} / k_{y}=k_{0} / \Delta k \simeq 2 f$. There is, however, no instability for supersonic flow $|M|=\left|v_{p, y} / c_{s}\right|>1$. In detail this has been worked out in Ref. 32 for the case of RPP smoothed laser beam. This work clearly shows via numerical simulations that PSF growth is enhanced for subsonic flow with respect to the case without flow $v_{p, y} \equiv 0$.

We have solved Eq. (12) as a function of $k_{y}$ for different values of the Mach number $M$ for subsonic flow, and for two cases with intensities $I_{\mathrm{L}} \lambda_{0}^{2}=10^{14} \mathrm{~W} / \mathrm{cm}^{2} \mu \mathrm{m}^{2}$ and $2.5 \times 10^{14} \mathrm{~W} / \mathrm{cm}^{2} \mu \mathrm{m}^{2}$, respectively (and for $n_{e} / n_{c}=0.1$, $T_{e}=3 \mathrm{keV}$, and $\hat{v}=0.05$ ). The unstable solutions found are shown in Figs. 4. For the case without flow, $M=0$, one observes a clear cutoff $k_{\text {cutoff }}$ in $k_{y}$ above which, $k_{y}>k_{\text {cutoff no }}$ unstable solutions exist. For the two cases shown, this cutoff corresponds to the threshold criteria for speckles with $I_{\mathrm{sp}}=I_{L}$ and $P_{\mathrm{sp}} / P_{c}=0.2$ and 0.5 , respectively, for $f=8$. In contrast to this, one cannot observe a cutoff for the subsonic flow cases, $0<|M|<1$. It is furthermore evident from the spatial growth values $q\left(k_{y}\right)$ obtained, that for subsonic flow the ponderomotive modifications expected from PSF are stronger than for $k_{y}<k_{\text {cutoff }}$ in the case without flow. The linear growth rate of the filamentation instability Eq. (12) has been discussed in Ref. 33 where it is shown that the enhanced density perturbations due to the instability contribute to the laser beam deflection. Such a beam bending has been observed in simulation results ${ }^{34,35}$ and can contribute to CBET.

Beam bending enhanced by the PSF of a single Gaussian beam is illustrated in Figure 5. In the case shown the beam is originally focussed at $x=1000 \lambda_{0}$. The plasma flow transverse to the propagation direction of the entering beam is a $M=0.96$. For the beam intensity chosen, the criterion according to Eq. (5) yields $P_{\mathrm{sp}} / P_{c}=0.5$, it is thus below the onset value for PSF. What can be seen shows, however, a clear onset of PSF and beam bending: the beam is deflected into a direction oblique with respect to its incidence, and the beam stays trapped in its own plasma density channel for distances considerably longer than the Rayleigh length. We have furthermore examined cases with still higher intensity values of single beams (not shown), for which strong non-stationary behavior can appear together with flow, driving density channels in the plasma that eventually propagate freely, leading to shock-like stuctures in the plasma density.

For crossing beams, it follows that the processes of beam bending and speckle self-focusing leads to an effective energy transfer into the other beam. For the described scenario of transfer between speckles and the other beam at close-to-sonic flow, one again has to consider the basic theory for CBET, a
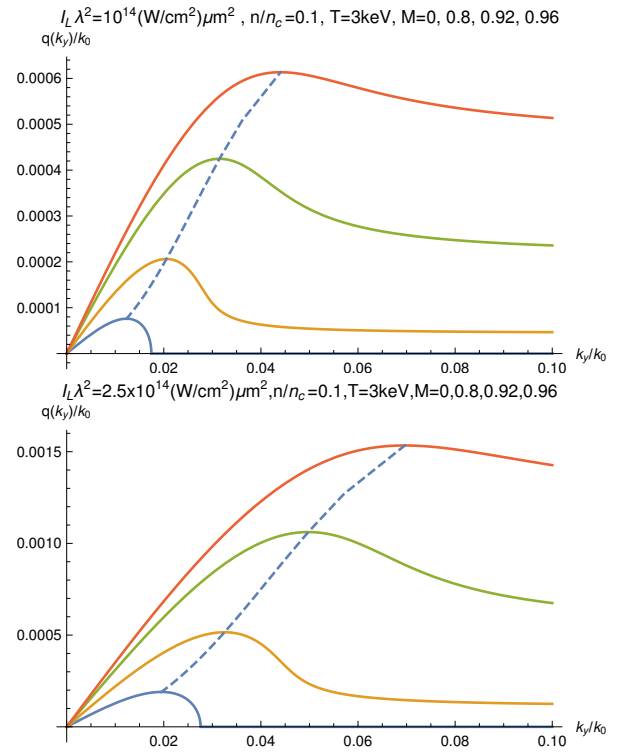

FIG. 4. Spatial growth rate $\Re q$ evaluated from Eq.(12) for the cases with intensities $I_{L} \lambda_{0}^{2}=10^{14} \mathrm{~W} / \mathrm{cm}^{2} \mu \mathrm{m}^{2}$ (upper plot) and $2.5 \times 10^{14} \mathrm{~W} / \mathrm{cm}^{2} \mu \mathrm{m}^{2}$ (lower) (and for $n_{e} / n_{c}=0.1, T_{e}=3 \mathrm{keV}$, and $\hat{v}=0.05)$, as a function of the transverse wave number $k_{y} / k_{0}$ and for different plasma (transverse) flow Mach numbers, $M=v_{p, y} / c_{s}=0$, $0.8,0.92$, and 0.96 . The dotted curves indicate the optimum growth rate as a function of $k_{y}$.

outlined before in Eqs.(7)-(11). Two differences essentially emerge as compared to 'beam-to-beam' CBET, namely:

(i) the speckle width, $f \lambda_{0} \sim D$, is most likely smaller than the inhomogeneity length, yielding $f \lambda_{0} / 2 \sin \theta<L_{\text {inh }}$, and

(ii) the local intensity inside a speckle can be several times (say, up to 6-9 times) higher than the average beam intensity. One can approximate $\left|a_{01}\right|^{2}\left|a_{02}\right|^{2}$ in Eqs. (7) by $\langle I\rangle I_{\mathrm{sp}} /\langle I\rangle^{2}$.

Consequently, the approximation for the gain describing the transfer between speckles and the other beam results in $G \simeq f \lambda_{0} \sin \theta \gamma_{0, \text { sp }}^{2} /\left(v_{s} c_{s}\right)$ in which $\gamma_{0, \text { sp }}$ is the SBS growth rate evaluated for the speckle intensity $I_{\mathrm{sp}}$. Compared to the gain values expected for 'beam-to-beam' CBET this gain value is by the factor $f \lambda_{0} / \min \left\{D /(2 \sin \theta), L_{\text {inh }}\right\}$ different. The width of a single speckle, $f \lambda_{0}$ is generally much smaller than the width of a RPP laser beam or the width of the interaction region in an inhomogeneous profile, whereas the factor $\gamma_{0, \mathrm{sp}}^{2}$ can assume values up to 6-9 times higher than $\gamma_{0}^{2}$, for the average laser beam.

Amplification of individual speckles by crossing overall beams has also been seen in experiments ${ }^{45}$. However, this transfer process into intense speckles remains transient as has been demonstrated in the experiments Ref. 45 involving relatively short laser pulses, of 2-4 ps duration. For longer laser 


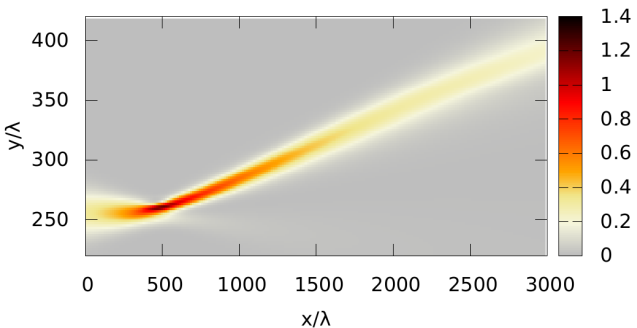

FIG. 5. Intensity contour map of an initially Gaussian beam, illustrating the deflection and the trapping of the beam in space $x$ and $y$, taken at an asymptotic stage, for the case of a plasma with transverse flow at $M=0.96$. The beam was initially focussed at $x=1000 \lambda_{0}$. Following the standard criterion of PSF for this case, the rbeam power to critical power ratio $P / P_{c}=0.5$ according to the criterion Eq. (5) without flow.

pulse durations the transfer from one to the other beam affects the whole beam. Transfer from speckles to the crossing overall beam is, on the other hand, very likely to happen, and is also seen in our simulations. The consequences of the latter process, as will be discussed and illustrated later on in section IV B, lead mostly to enhancement in the angular spread of the beam receiving energy from speckles.

\section{Density perturbation and beam bending}

Although the steepening of the ion acoustic waves is a nonlinear process, the linear approximation of the plasma response in presence of background flow already clearly points to the shock formation, which is well reproduced in our simulations. It is helpful to recall a model of the plasma response to the stationary ponderomotive potential in the presence of transverse flow. The linear response of the plasma density is described by the wave equation obtained from linearizing Eqs.(3a)-(3b),

$$
\left(\partial_{t}^{2}+2 v_{s} \partial_{t}-c_{s}^{2} \partial_{y}^{2}\right) \delta n / n_{e}=c_{s e}^{2} \partial_{y}^{2} U
$$

where $\delta n / n_{e}$ is the density perturbation, $v_{s}$ the IAW damping; $U$ denotes in this section the normalized ponderomotive potential, $U \equiv U_{\text {self }} / T_{e}$. A convenient way to introduce a transverse flow into Eq. (13) is to consider a moving beam in a stationary plasma ${ }^{35}$ that is equivalent to the flowing plasma in $y$-direction and a stationary laser beam, or crossed beams giving rise to the ponderomotive potential. In this new frame of reference $U=U_{\text {self }}\left(y+v_{p, y 0} t\right)$ describes the ponderomotive potential moving to the left with a uniform plasma flow velocity $v_{y 0}$. The analytical solution to Eq. (13) can be obtained using the procedure of Ref. 57, where we introduced the Laplace transform of $\delta n / n_{e}$ and of $U,\{\delta \tilde{N}, \tilde{U}\}(y, s)=$ $\int_{0}^{\infty} d t e^{-s t}\left\{\delta n / n_{e}, U\right\}(y, s) ; \delta \tilde{N}$ satisfies the wave equation,

$$
\left(\partial_{y}^{2}-\beta^{2}\right) \delta \tilde{N}=-\partial_{y}^{2} \tilde{U}(y, s)
$$

for $c_{s} \simeq c_{s e}$ with $\beta^{2}=\left(s^{2}+2 v_{s} s\right) / c_{s}^{2}$, having the solution

$$
\delta \tilde{N}(y, s)=\frac{e^{-\beta y}}{2 \beta} \int_{-\infty}^{y} d \xi e^{\beta \xi} \partial_{\xi}^{2} \tilde{U}-\frac{e^{\beta y}}{2 \beta} \int_{\infty}^{y} d \xi e^{-\beta \xi} \partial_{\xi}^{2} \tilde{U} .
$$

After integration by parts and calculating the inverse Laplace transform, $\delta N(t)=1 /(2 \pi i) \int_{-i \infty+\sigma}^{i \infty+\sigma} d s e^{s t} \delta \tilde{N}(s)$, we obtain the following time dependent solutions for the density perturbation in the frame of the moving plasma and for the stationary ponderomotive potential: for plasma flow different from the speed of sound, i.e. for $M \equiv v_{y 0} / c_{s} \neq 1$, one obtains

$$
\begin{aligned}
\delta N(y, t) & =-\frac{U(y)}{1-M^{2}}+\frac{v_{s}}{2 c_{s}}\left[\frac{I^{(+)}}{1-M}+\frac{I^{(-)}}{1+M}\right] \\
& +\frac{1}{2} e^{-v_{s} t}\left[\frac{U\left(y+(1-M) c_{s} t\right)}{1-M}+\frac{U\left(y-(1+M) c_{s} t\right)}{1+M}\right],
\end{aligned}
$$

with $I^{( \pm)}=\int_{0}^{c_{s} t} d \xi e^{-v_{s} \xi / c_{s}} U(y \pm \xi-M \xi)$ and with the approximation $\beta \approx\left(s+v_{s}\right) / c_{s}$. For sonic plasma flow, $M=1$, the density response from Eq. (15) yields the expression,

$\delta N(y, t)=\frac{1-e^{-v_{s} t}}{2} \frac{\partial_{y} U(y)}{v_{s} / c_{s}}-\frac{U(y)}{2}+\frac{U\left(y-2 c_{s} t\right)}{4} e^{-v_{s} t}+\frac{v_{s} I^{(-)}}{4 c_{s}}$.

In Figures 6-7 we show the density perturbation response to the ponderomotive force for the case of a speckle in the $y$ direction transverse to the laser propagation, close to focus, taken at late times $v_{s} t \gg 1$. Figure 6 illustrates, for different Mach numbers, $M=0.9,1$, and 1.1, the linear response computed from the model Eq. (16) to a Gaussian-shaped speckle, $U(y)=U_{0} \exp \left\{-y^{2} /\left(f \lambda_{0}\right)^{2}\right\}$, namely $\left(n-n_{0}\right) / U_{0}=$ $\delta N(y, t) / U_{0}$, where $n_{0}$ corresponds to the equilibrium density and $U_{0}$ to the peak value of the ponderomotive force in the center of a laser speckle. The solid and dashed curves correspond to the damping rates $v_{s}$ with $\left(v_{s} / c_{s}\right)\left(f \lambda_{0}\right)=0.15$ and 0.05 , respectively. For the lower damping rate the asymmetry predicted from Eq. (16) is more pronounced. Note that for the sonic case $M=1$, for which the 2 nd term $\sim \partial_{y} U(y)$ in Eq. (17) dominates (because of $\left(c_{s} / v_{s}\right) f \lambda_{0}>1$ ), the density perturbations reach the highest amplitudes and display the steepest gradients. The asymmetry between maximum and minimum of density response contributes to the beam bending ${ }^{33,35}$ and modifies CBET in our simulations.

Figure 7 shows the response obtained from simulations, for $M=0.9,1$, and 1.1, accounting also for non-linearity in Eqs. (3a)-(3b). The gray curve shows the case when the speckle power is well below PSF critical power (according to Eq. (5)), $P / P_{c}=0.05$ for $M=1$, the other curves show the case with $P / P_{c}=1$, where a departure from the linear response becomes visible mostly in the loss of symmetry between $n(y)-n_{0}>0$ and the density depression $n(y)-n_{0}<0$ which is deeper but also more localized. The corresponding spectra of $n-n_{0}$ as a function of $k_{y}$ are shown in Fig. 8: the Gaussian-shaped form as a function of $k_{y}$ is preserved only in the linear regime, with a peak around $k_{y} \simeq k_{0} / f$. For the non linear case, here with $P / P_{c}=1$, the peak in the spectrum is found at lower $k_{y}$ values, almost $k_{y} \simeq k_{0} /(2 f)$ with a linear (Lorentzian-type) exponential decrease. We shall see later that these spectra help 


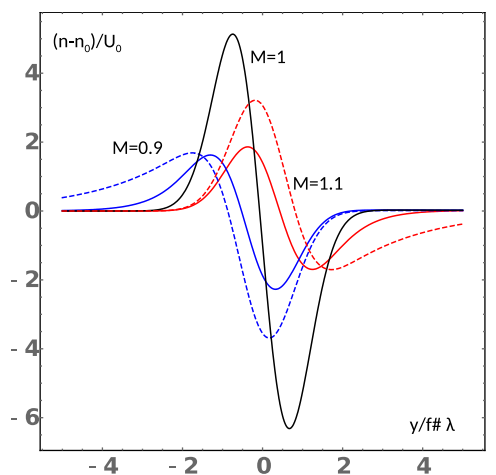

FIG. 6. Density perturbation response as a function of $y$ according to Eq. (16) to a Gaussian-shaped laser speckle $U(y)=$ $U_{0} \exp \left\{-y^{2} /\left(f \lambda_{0}\right)^{2}\right\}$ for 3 different flow Mach numbers, $M=0.9$ (blue curves), 1 (black), and 1.1 (red), and for 2 different damping rates, $\left(v_{s} / c_{s}\right)\left(f \lambda_{0}\right)=0.15$ (solid lines) and 0.05 (dashed lines).

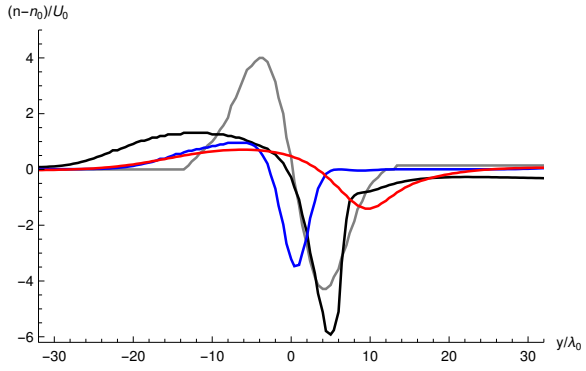

FIG. 7. Density perturbation response as a function of $y$ from simulations of a laser speckle at focus, having a Gaussian shape as boundary condition at $x=0$, same parameters as in Fig. 6, taken at focus (in $x$ ). The gray curve shows a low-intensity case, $P / P_{c}=0.05$ for $M=1$. The other cases, for $P / P_{c}=1$, show non linear respnse with self-focusing, for 3 different flow Mach numbers, $M=0.9$ (blue curves), 1 (black), and 1.1 (red). In all cases the damping rate is $\left(v_{s} / c_{s}\right)\left(f \lambda_{0}\right)=0.15$

to partially interpret the ion density perturbations in a multispeckle environment of crossed beams.

\section{ANALYSIS OF THE SIMULATION RESULTS}

In the present study, for all simulations in Figs. 2-3,9-11, we have chosen a domain of $4500 \lambda_{0}$ in length and $2300 \lambda_{0}$ in width, i.e. along the $x$ and the $y$ axes, respectively; the beams have a common wave vector component along $x$ and opposite wave vector components along $y$. In this chosen geometry, the gradients of the plasma profiles in density and velocity point predominantly along the $y$-direction. The density profile is parabolic around the center, $y=L_{y} / 2$, given

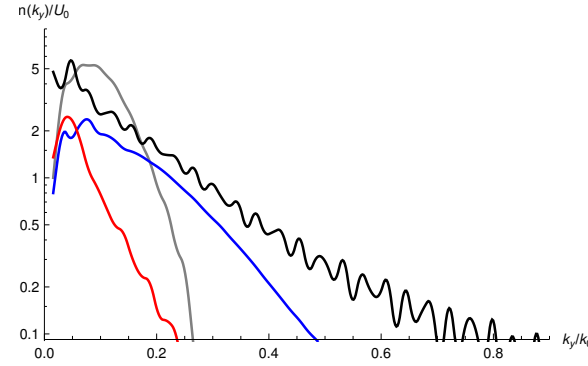

FIG. 8. Fourier spectrum of the density perturbation response as a function of $k_{y}$ from simulations with a Gaussian laser speckle at focus, corresponding to Fig. 7, namely for a low-intensity case $\left(P / P_{c}=0.05\right.$, gray curve $)$ with $M=1$, and for $P / P_{c}=1$, corresponding to the non linear cases at 3 different flow Mach numbers, $M=0.9$ (blue curves), 1 (black), and 1.1 (red).

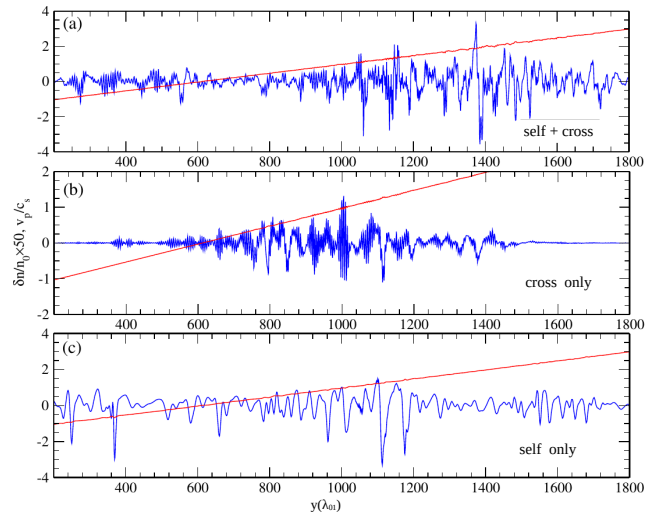

FIG. 9. Density imprint of two crossing RPP beams as a function of $y$ taken at $x / \lambda_{0}=1500$ in the front part of the rhombus-shaped crossing zone. Subplots (a) shows the density perturbation when both crossedbeam coupling ('cross') via $\nabla U_{\text {cross }}$ and beam self-interaction via the $\nabla U_{\text {self }}$ term ('self') are taken into account in HARMONY simulations, the other subplots show the case when only the $\nabla U_{\text {cross }}$ term (b) or only the $\nabla U_{\text {self }}$ term (c), respectively, are taken into account. The shown results correspond to the same parameters as in the cases of RPP in Fig. 2. The red line shows the flow profile $v_{p, y}(y) / c_{s}$ with a sonic point at $y \simeq 1100 \lambda_{0}$.

by $n_{e}(y)=0.1 n_{c} \exp -\left[\left(y-L_{y} / 2\right) / 1615 \lambda_{0}\right]^{2}$. We apply a linear density ramp starting at $x=0$ over $500 \lambda_{0}$ along $x$ in order to avoid boundary effects at the laser entry. As already mentioned earlier in section II we apply a linear flow ramp with sonic flow in the center of the crossing beams, i.e. $v_{p y}\left(y=L_{y} / 2\right)=+c_{s}$, and a flow gradient $L_{v}=200 \lambda_{0}$. We have focused our study on the case when both beams have equal intensity $I_{01}=I_{02}$ at the entrance $x=0$, and both 

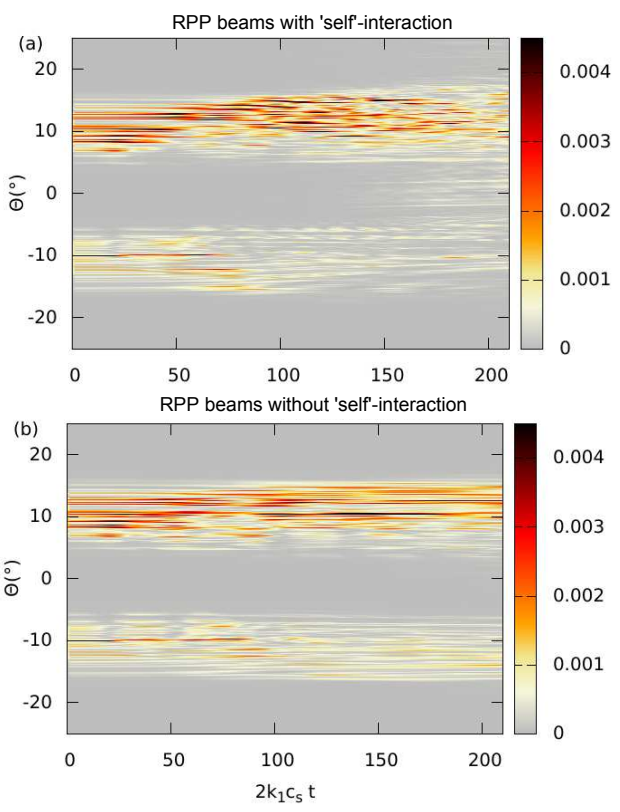

FIG. 10. The subplots (a) and (b) respectively show, as a function of time, the angular spectrum of the transmitted light, computed on the basis of a single RPP realization. The values are taken from fields at the rear of the simulation box for RPP beams in Fig. 2, in presence (a) and in absence (b) of the $\nabla U_{\text {self }}$ term in Eq. (3b). The upper part, for angles $>0$, corresponds to beam 2 , the lower part to beam 1 .

beams have the same focusing $f$-number, namely $f=6$ for RPP beams. Here the coefficient $\hat{v}$ takes the value $\hat{v}=0.1$, except in the study examining sensitivity of CBET on the ion acoustic wave damping, see section IV B. In plasmas with inhomogeneous flow, CBET occurs when the effective beam width, $L_{\text {beam }}=D /(2 \sin \theta)$ is larger than the interaction length $L_{\text {inh }}=\pi\left[v\left(k_{s}\right) / \omega_{s}\right]\left[L_{v} / \sin (\theta / 2)\right]$, where D is the beam diameter ${ }^{19}$. This is equivalent to $D / L_{v}>4 \pi\left(v / \omega_{s}\right) \sin (\theta / 2)$, in practical units $D / L_{v} \sim .2\left(\theta / 20^{\circ}\right)\left(v / 0.1 \omega_{s}\right)$ for small $\theta$.

\section{A. The role of speckles and ponderomotive self-focusing on CBET in RPP and regular beams}

In order to illustrate the role of laser speckles and of ponderomotive self-focusing on CBET in presence of a flow, we compare simulation results between the cases of (i) two crossed RPP beams and of (ii) two 'regular' beams. The regular beams have the same average intensities and envelope shapes as the RPP beams.

In the following we illustrate our results in two subsections: in the first one we show results of interacting laser beams based on a single realization of a RPP, in the second subsection, section IV A 2 and the Figs. 3 and 11, we show results based on the ensemble average over eight realizations of RPP. No ensemble averaging is necessary for 'regular' beams.

\section{Results from a single realization of RPP beams}

All snapshots shown in Figs. 2(a)-(c) are based on a single RPP realization for each beam; they illustrate the local dynamics arising due to the speckles of this realization. Figs. 2(d)-(f) are based on regular beams. Figures 2(a) and 2(d) show the initial $(\mathrm{t}=0)$ beam geometry before interaction, for the cases of two crossed RPP and of two 'regular' beams, respectively. One may again consult Fig. 1 for the general geometry of the simulations. We display the particular case where the average beam intensities are $I_{01}=I_{02}=6 I_{0}$. The interaction region of the two beams for both cases forms a rhombus-like shape in the center of the simulation box. In our configuration, the plasma flow profile is chosen in a way that sonic flow appears along the major diagonal of the rhombus, which is parallel to the longitudinal direction $x$. CBET is hence excited around $y=L_{y} / 2$ with a plasma flow gradient $\propto 1 / L_{v}$ along the $y$-axis.

\section{Crossing speckle beams: PSF and flow}

Figure 2(b) shows that in the presence of self-interaction, the two crossed RPP beams undergo significant deflections with respect to the initial beam directions (see Fig. 2(a)); also the angular aperture of each beam is broader than initially, while without the $\nabla U_{\text {self }}$ force, see Fig. 2(c), the beams transfer energy without significant deflection or broadening. Figure 2(b) exhibits also features of plasma induced smoothing ${ }^{54}$ and moving filaments ${ }^{58}$ at the rear of the simulation box. The two terms $\nabla U_{\text {self }}$ and $\nabla U_{\text {cross }}$ in Eq. (3b) are responsible for this additional spatial and temporal incoherence in the transmitted light. In Fig. 2(b), an enhanced transfer of energy from beam 1 to 2 (as will be shown later) and a strong angular spread in presence of self-interaction are observed. These processes are due to the fact that for $I_{01}=I_{02}=6 I_{0}$ a significant population of speckles has sufficiently high power to be unstable with respect to PSF. As elaborated in section III B, due to flow, PSF in speckles occurs already at intensities lower than indicated by the standard expression Eq.(5) for $P_{s p} / P_{c}$.

Our simulation results demonstrate the importance of the plasma flow. For an inhomogeneous flow profile, as in our simulations, resonant coupling between the crossing beams takes place around the region where the plasma velocity is close to the sound velocity, $v_{p, y} \sim c_{s}$. This is where one can see in Fig. 2(e) that beam bending arises so that some speckles are redirected towards the other beam and effectively contribute to CBET

\section{Crossing regular beams: PSF and flow}

Regular beams, because of the flat, almost plane wave-type wave fronts can be unstable to PSF and to the filamentation instability for the intensity range considered, so that any perturbation in the beam structure or in the plasma density will trigger the onset of filamentation instability. Such initial perturbations in regular beams are produced by the ponderomotive force of crossing beams. They can further develop and 
lead to filamentation in the simulations when the $\nabla U_{\text {self }}$ term is taken into account. The interaction of two crossed regular beams illustrated in Fig. 2(e) (with both the $\nabla U_{\text {self }}$ and the $\nabla U_{\text {cross }}$ terms taken into account) results in the transfer of energy into a beam that propagates along a common axis. Filamentary structure develops in the overlapping regular beams where they interact with the density modulations due to the ponderomotive forces induced by the crossing beams. The regular beam filaments also undergo beam bending as seen in Fig. 2(e) at near to sonic flow, $v_{p}= \pm c_{s},{ }^{34,59}$. This contributes to the beam component propagating along $\mathrm{x}$-axis, and partially to angular broadening of the transmitted light. We have also carried out a simulation without applying the $\nabla U_{\text {self }}$ term, see Fig.2(f). For this case, perturbations in both beams arise inside the (rhombus-shaped) resonant zone for CBET, and - in contrast to the case with self-interaction - no further filamentation develops in the small beamlets. Note that the structures of regular beams induced by CBET and by filamentation point mostly along the common axis between both beams, a feature that is clearly not observed in the RPP beams with speckle structure.

\section{Induced density perturbations}

For the case of crossing RPP beams, significantly different density perturbations are excited in the plasma when taking into account both the effects of ponderomotive self-interaction and crossed-beam coupling together ('self + cross' in Fig. 9(a) ), or only a single one of these effects, i.e. only crossedbeam coupling ('cross only' in Fig. 9(b) ) or only beam selfinteraction ('self only'), respectively. The latter is illustrated via a the density perturbations defined as $\delta n \equiv n(x, y, t)-n_{e}$ with $n_{e} \equiv n(x, y, t=0)$, taken at $x \simeq 1500 \lambda_{0}$ in the front part of the rhombus, and shown in the three subplots of Fig. 9 , also indicating the flow profile $v_{p, y}(y) / c_{s}$, with sonic flow at $y / \lambda_{0}=1100$.

\section{Angular broadening of the beams}

In addition, and in order to illustrate the effect of deflection and angular broadening observed for crossed RPP beams in Figs. 2(b) (in presence of beam self-interaction) and 2(c) (in absence of beam self-interaction), we plot the temporal evolution of the angular spectrum of the transmitted light (detected at the rear of the simulation box) in Figs. 10(a) and 10(b), respectively. The light signals appearing in the upper right corner of the simulation box between time $2 k_{1} c_{s} t=150$ and 200 in Fig.10(a), show that in presence of self-interaction, beam 2 - initially propagating at an angle of $10^{\circ} \pm 3.5^{\circ}$ - has components up to large angles of $\sim 25^{\circ}$, with a central direction at $11^{\circ}$ ( width $\pm 7^{\circ}$ ), while in absence of the $\nabla U_{\text {self }}$ term, Fig. 10(b), beam 2 does not undergo strong deflection: it is simply characterized by an asymmetric angular spread around $\sim 11.5^{\circ}\left(+5^{\circ} /-4^{\circ}\right)$, at $t=200$. Similarly, beam 1 is characterized by an enhanced angular broadening around $-10^{\circ}\left( \pm 5.5^{\circ}\right)$ in the case with beam self-interaction (Fig. 10(a) ) by contrast with the case without beam self-interaction (Fig. 10(b) ), showing an asymmetry around $-12^{\circ}\left(-5^{\circ} /+4^{\circ}\right)$; the latter is interpreted as due to pump depletion.

To summarize these results, as seen in Figs. 2 and 10, one can characterize the role of speckle structure for CBET as fol- lows: (i) the importance of speckle structure for CBET increases with beam intensity due to the increasing number of speckles undergoing PSF; (ii) the onset of PSF in subsonic flow regions occurs in speckles with peak intensities lower than predicted by the standard criterion, Eq. (5), which eventually increases considerably the number of speckles affected by PSF; (iii) at sonic flow, speckle beams are deviated by beam bending which can lead to a net transfer into the other beam. (iv) Particularly striking is the onset of temporal incoherence of the beams for the case with self-interaction around $+10^{\circ}$, resulting in fluctuations with relatively short correlation times of $2 k_{1} c_{s} t_{\text {corr }} \sim 20$ ( $t_{\text {corr }} \sim p s$ in real units), that can be associated to the effect of plasma-induced smoothing.

\section{Ensemble averaging over multiple RPP realizations}

In the following we will revisit the previous results and analyze them further by both varying the beam intensity as well as by examining the angular aperture of the transmitted beams. To do so, we have averaged results of simulations over different RPP realizations. In Figure 3 we have summarized our results from a series of simulations with RPP beams; we have averaged over eight realizations. Shown is the energy transfer as a function $I_{02} / I_{0}$, which clearly shows that the role of RPP speckles in CBET can no longer be disregarded above the reference intensity, $I_{0} \lambda_{0}^{2}=10^{14} \mathrm{~W} / \mathrm{cm}^{2} \mu \mathrm{m}^{2}$ at $3 \mathrm{keV}$. To illustrate this we plot the transfer from beam 1 (downwards propagating) to beam 2 by increasing the incoming intensity values of both $I_{01}$ and $I_{02}$, while keeping their ratio $I_{01} / I_{02}=1$ constant. We compare the power gained by beam 2 for the RPP (subplot (a), solid curves) and regular (subplot(b), dashed curves) beam cases in presence (blue curves) and absence (red curves) of the $\nabla U_{\text {self }}$ terms. To do so in the simulations with HARMONY, we switched on and off this term on the r.h.s. of Eq. $3 b$. The power transfer ratio is defined as

$$
\frac{P_{\text {out }}}{P_{\text {in }}} \equiv \frac{\int_{k_{y}>0}\left|\mathscr{E}\left(k, x=L_{x}\right)\right|^{2} d k}{\int_{k_{y}>0}|\mathscr{E}(k, x=0)|^{2} d k},
$$

where $\mathscr{E}\left(k_{y}\right)$ is the 1D Fourier transform of $a(x, y, t)$ in $y$.

Note that Fig. 3(b) also displays a curve (in green color) that corresponds to the results from Ref. 19 for the interval $0<I_{02} \lesssim 0.75 I_{0}$, for the same geometry, also obtained using our code HARMONY. In this interval no significant differences in the transfer between the beams was seen when comparing regular and RPP beams, even when accounting for selfinteraction. The differences seen between the results for different RPP realizations are merely due to speckle statistics ${ }^{28}$.

From our new results, differences due to PSF in speckles appear for $I_{02} \gtrsim 0.75 I_{0}$, when one enters in the regime where ponderomotive effects as self-channeling, deflection via beam bending in a flowing plasma, as well as plasmainduced smoothing occur. For RPP beams all these processes depend on the laser speckle distribution.

We should mention here that experimental studies with crossed RPP beams ${ }^{17}$ have reported both on angular broadening and on spectral broadening in an intensity regime between $2.4-8 \times 10^{13} \mathrm{~W} / \mathrm{cm}^{2}$ at $\lambda_{0}=1 \mu \mathrm{m}$, however at lower temperature $(0.5 \mathrm{keV})$ and higher density $\left(0.3 n_{c}\right)$ than considered in 

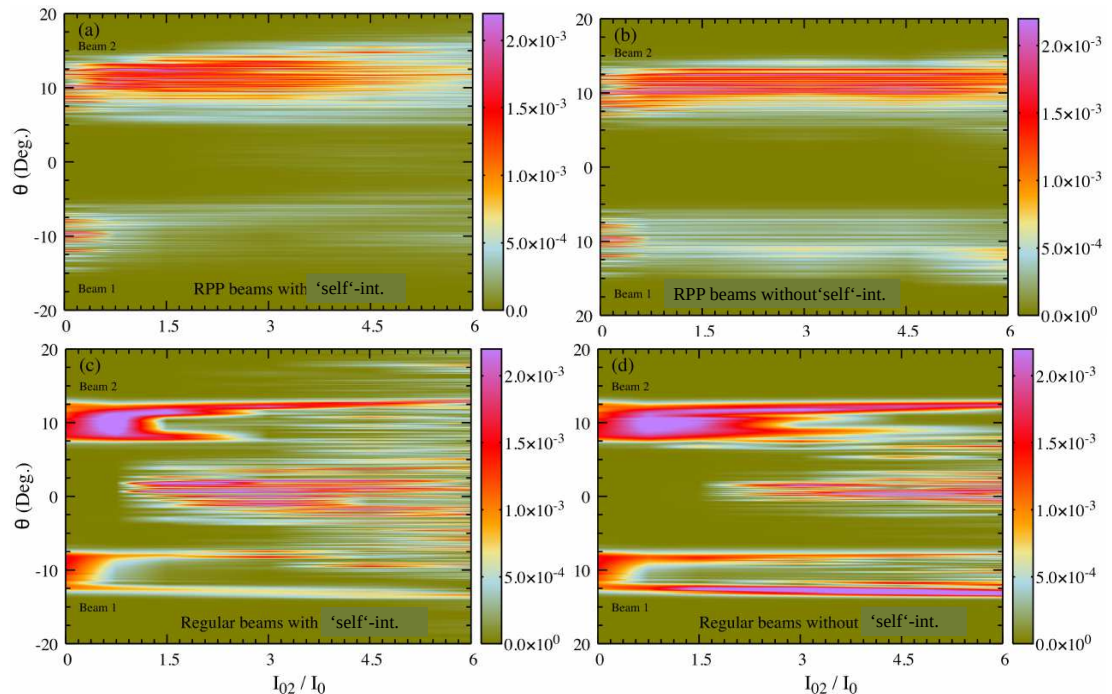

FIG. 11. Contour plots of angular spectra of the transmitted light, as a function of the incident intensity $I_{02} / I_{0}$ for equal beam power at the input, $I_{01}=I_{02}$, and deduced from the fields taken at the right edge of the interaction region at $t=200$. Panels a) and $\mathrm{b}$ ) in the upper row show the cases of RPP beams (average over 9 realizations), panels c) and d) show regular beam cases. The left column, panels a) and c), shows the behavior with beam self-interaction via $\nabla U_{\text {self }}$, while in the right column, panels b) and d), beam self-interaction is not taken into account. Note: the color bar shows values normalized to the total power $\left(\propto I_{02}\right)$ of the corresponding beam intensity $I_{02}$. Contours are interpolated from five distinct values at $I_{02} / I_{0}=0.75,1.5,3,4.5$, and 6 .

\section{this article, with effects of collisional absorption present.}

\section{The role of self-focusing in speckles}

Our simulation results summarized in Fig. 3 show as a function the the beam intensity, that for $I_{02} / I_{0}>0.75$, the power transfer to beam 2 first increases for the case of RPP beams with self-interaction (Fig. 3(a) solid blue curve), then reaches a maximum around $I_{02}=I_{01}=3 I_{0}$, and eventually decreases for still higher intensities. There is a striking difference between the cases with and without the effect of selfinteraction : when neglecting the self-interaction effects, no significant increase in power transfer as a function of $I_{02}$ is seen, and the onset of the decrease in the power transfer occurs already for $I_{02}=I_{01} \gtrsim 1.5 I_{0}$. Although the standard threshold criterion for PSF in Eq. (5) would indicate that only few extreme speckles can have $P_{\mathrm{sp}} / P_{c}>1$, it is the presence of flow that changes considerably the PSF in speckles in the beam overlapping region, both for sonic and subsonic flows. Consequently, the light is deflected towards the direction of beam 2, which is a net contribution to CBET for RPP beams in the intermediate regime $1 \lesssim I_{02} / I_{0} \lesssim 3$. Also, for $I_{02} / I_{0}>0.75$, comparing the blue and red curves in Fig. 3(a), with and without the self-interaction effects, respectively as expected - it can be clearly seen that the power transfer is systematically stronger when the contribution of the selfinteraction effects in the ponderomotive force is taken into ac- count. The relevant regime corresponds, in practical units, and for the plasma conditions considered here, to laser fluxes from $I_{02} \lambda_{0}^{2}>10^{14} \mathrm{~W} \mu m^{2} / \mathrm{cm}^{2}$. This means that onset of PSF effects in speckles arises still in an intensity regime that is very relevant with respect to current ICF parameters.

The power transfer between regular beams, shown in Fig. 3(b) for $I_{02} / I_{0}>0.75$, is also systematically lower than for the power transfer between RPP beams. For regular beams (dashed curves), without speckles, the self-focusing and forward SBS play only a limited role, in spite of the differences in the angular spectra observed in Fig. 11(c) and (d) for the cases with (c) and without (d) self-interaction effects. For high beam intensities, $I_{02} / I_{0}>2$, one reaches a maximum power transfer between the beams, beyond which the power transfer decreases as a function of $I_{02}$. This is due to the nonlinearities of the ion acoustic wave perturbations (see next section). Nonlinear sound waves enhance forward scattering and diffraction to a broader angular spread of the transmitted beams, which yields asymptotically an effective equilibration between the angular spectra of both beams towards $\int_{k_{y}>0}\left|\hat{a}\left(k, x=L_{x}\right)\right|^{2} d k \simeq \int_{k_{y} \leq 0}\left|\hat{a}\left(k, x=L_{x}\right)\right|^{2} d k$.

\section{Angular broadening as a function of intensity}

The observed broadening of the angular spectrum, as displayed as a function of time in Fig. 10 for the single example of a RPP beam with $I_{01}=I_{02}=6 I_{0}$, is summarized as a func- 


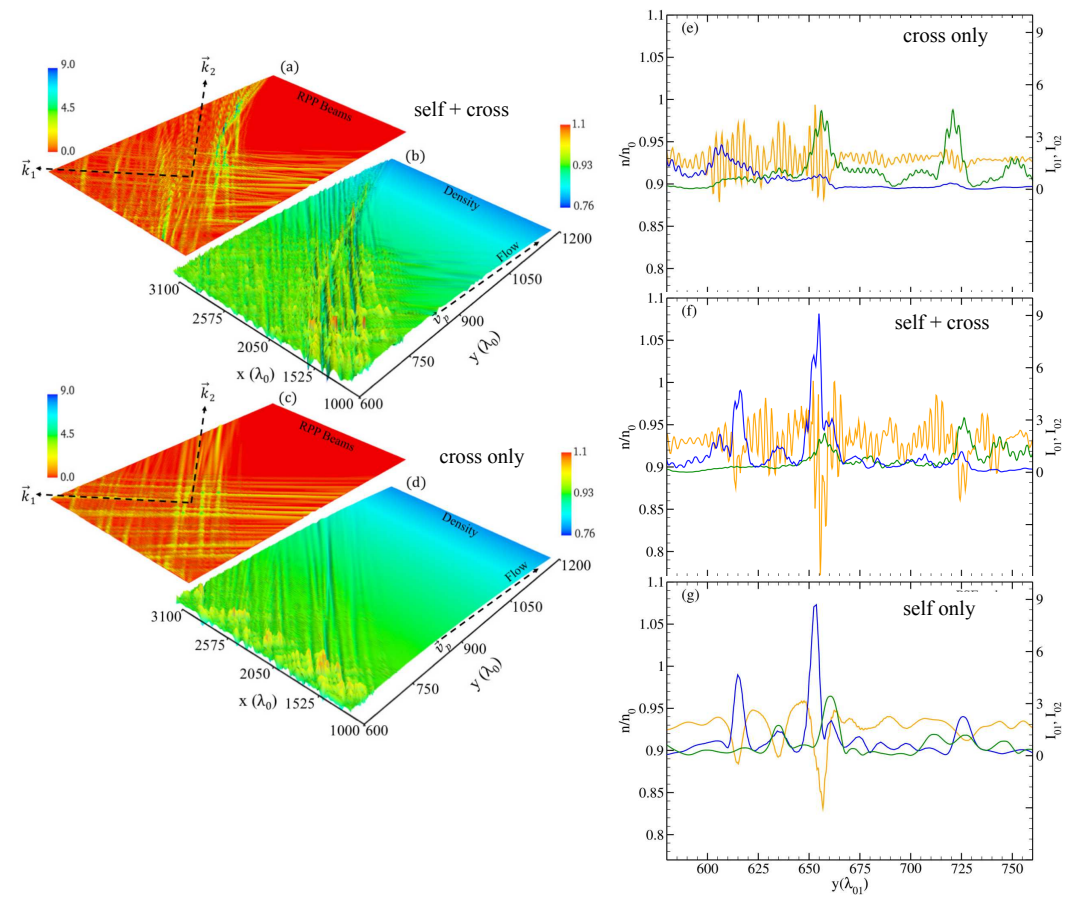

FIG. 12. (Color online) Simulation results showing beam deflection and shock generation: (a)-(d) show the zoomed in regions of interaction between the two crossed RPP beams of the same intensity $I_{01}=I_{02}=6 I_{0}$, crossing at an angle $\theta=20^{\circ}$ and $\hat{v}=10 \%$. Subplots (a)-(d) respectively show a snapshot of the beam intensity (a),(b) and the perturbed plasma density (c),(d) taken at $t=200\left(2 k_{1} c_{s}\right)^{-1}(\sim 11 \mathrm{ps}$ for $\lambda_{0}=0.35 \mu \mathrm{m}$ and $T_{e}=3 \mathrm{keV}$ ) in presence of both self-interaction and crossed-beam coupling. In subplots (a) and (b) both processes are taken into account, in panels (c) and (d) only crossed-beam coupling. The figures (e)-(f), are lineouts (along $y$ and at $x=1050 \lambda_{01}$ in (a)-(d)) for the three different cases (e) crossed-beam coupling only, (f) both self-interaction and crossed-beam coupling, and (g) self-interaction only. The orange curve shows the density profile, blue curves and green curves show the Intensity profiles of the two RPP beams.

tion of $I_{02} / I_{0}$ in Fig. 11. The values shown are taken late in time, namely $2 k_{1} c_{s} t=200$ when the CBET processes have reached a quasi-stationary regime. The comparison between the angular distribution of the transmitted light of the two crossed beams are shown for four different cases: Figs.11(a) and 11(b) show the cases of RPP beams, in presence and in absence of self-interaction, respectively; the Figs. 11(c) and 11(d) show the angular distributions of the transmitted ligh for regular beams. Generally, RPP beams clearly exhibit an increasing angular spread of transmitted light with increasing intensity; in addition, this angular spread is enhanced by selfinteraction. The results for regular beams (see Figs. 11(c) and $11(\mathrm{~d})$ ) are dominated by the strong central beam structure close to $\theta \sim 0$. The angular width of this central beam increases with $I_{02} / I_{0}$.

Two distinct beams can always be identified in the RPP case; only for the case of RPP beams with self-interaction weak beam arises around $\theta \sim 0$. The resulting angular spectra for RPP and regular beams start to broaden for $I_{02}>I_{0}$ shown in Figs. 11(a) with self-interaction. The case without self-interaction is shown in 11(b) with less pronounced broadening. Furthermore, the onset of angular spread contributes to the increase in spatial and temporal incoherence (see also 2(b) and Fig. 10(b)).

B. Nonlinear sound wave perturbations with self-interaction and their role in CBET

As already mentioned, the energy transfer between the crossing beams decreases according to Fig. 3 as a function of the beam intensity for $\mathrm{I}_{02} / \mathrm{I}_{0}>3$. This behaviour is correlated with both (i) the increase in the angular spread as a function of intensity, associated with enhanced spatial and temporal incoherence in each beam with increasing beam power, and with (ii) the onset of non- linearities in the IAW perturbations. Thus, it is important to analyze the role of nonlinear density perturbations in the plasma during CBET. For this purpose, we present in Figs. 12-14 a set of simulation results for the same laser-plasma parameters as used in Fig.2, however now with different realizations and a smaller simulation domain (with 

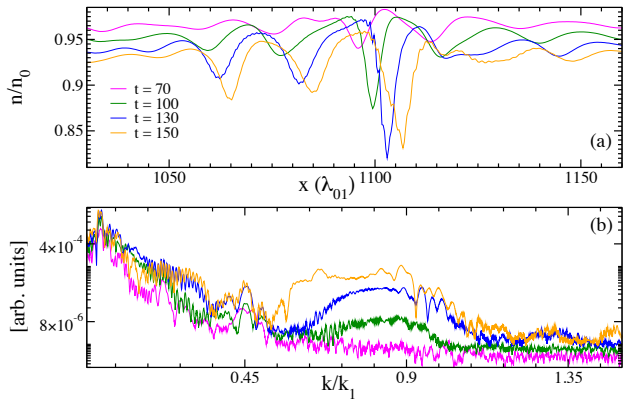

FIG. 13. (Color online) (a): Lineouts of the perturbed plasma density (along $y$ and at $x=1525 \lambda_{01}$ in Fig. 12(a)) for the self-interaction only case, taken at 4 different time instants, $2 k_{1} c_{s} t=70,100,130$, and 150. Panel(b) shows the spatial Fourier spectra in $k_{y} / k_{1}$ computed from the lineouts shown in panel (a) at the 4 different time instants.

a length of $3500 \lambda_{0}$ and $1200 \lambda_{0}$ along the $x$ (longitudinal) and $y$ (transverse) axes respectively). Figures 12(a) and (b) show the intensity profile and the IAW perturbations in the plasma, respectively, zoomed in the regions of overlap of the two crossed RPP beams. For the case shown both self-interaction and crossed-beam coupling are taken into account. Figures 12(c) and (d), show the same quantities as subplots 12(a) and (b) for the case where only crossed-beam coupling is taken into account while self-interaction is switched off. Comparing Figs. 12(a) and (c), we clearly see strong deflection of the RPP beams in the presence of self-interaction process while no deflections are observed in the absence of self-interaction. Also, comparing the corresponding density perturbations in the Figs. 12(b) and (d), one can observe nonlinear density perturbations (in terms of deep density channels) only for the case in the presence of self-interaction. In order to demonstrate this effect in detail, we also plot the line outs of the intensities of the two crossed RPP beams and of the plasma density perturbations for the three different cases in the set of Figs. 12(e)(f). These line outs are taken at $x \approx 1525 \lambda_{0}$ in the longitudinal direction and along the transverse direction $y$ from the fields shown in Figs. 12(a) and (c). In these figures of line outs the blue and green curves distinguish the intensity profiles of the two crossed RPP beams, while the orange curve shows the density profile. In Figs. 12(e), we can observe the dominant short wavelength plasma density perturbations (orange curve), having the wave length $\lambda_{\text {cbet }}=2 \pi /\left|k_{1, y}\right|$. The oscillations are seen in a wide zone around the resonant sonic flow region due to the IAWs in the pure CBET-SBS case; the blue and orange curves show the redistribution of energy between the two RPP beams. The low amplitude oscillations in density (orange curve in Fig. 12(e)) correspond to CBET between the average RPP beams, similarly to what would be seen in regular beams. We can also see regions with locally higher oscillations amplitudes corresponding to CBET where the exchange is enhanced between the average beam and intense laser speckles in the other beam. Figure 12(f) clearly shows the impact of PSF process in speckles along with the short-wavelength CBET-
SBS-driven IAWs. From the figure we see that redistribution of energy between the two beams under the combined effect of self-interaction and crossed-beam coupling leads to significantly higher intensity peaks as compared to the case with only crossed-beam coupling (Fig.12(e)).

For the case without the CBET-SBS process, as shown in Fig. 12(g), short-wavelength components are absent and the density perturbations are merely due the imprint of the ponderomotive force from the speckles. In the set of Figs. 12, sonic flow, i. e. $M=1$, is located in the vicinity of $y=655 \lambda_{0}$ for $x>600 \lambda_{0}$, where one can observe a phase shift between the induced density minimum and the intensity maxima. Outside the region of sonic flow, for $y<645 \lambda_{0}$ and $y>665 \lambda_{0}$, one can systematically observe that significant intensity maxima coincide spatially with density minima, as expected for ponderomotively induced density perturbations.

The two ponderomotive force terms on the r.h.s of Eq. (3b) can be strong enough to induce nonlinear density perturbations (as seen in Figs. 12(b) and (f)) in plasma with steepened wave fronts, as discussed earlier in section III B. Also, the plasma has a flow which affects the evolution of the density channels due to the PSF, and the IAW perturbations due to the CBET-SBS process, evolve in shock-like structures, characterized here by ponderomotively driven density perturbation that develop steep wave fronts when propagating in the plasma. The time evolution of such a shock-like structures is illustrated in Fig. 13(a). Plasma density perturbations are recorded along the same lineout (as in Figs. 12(e)-(f)) for different time instants and for a simulation case where only the $\nabla U_{\text {self }}$ term was retained. Also, Fig. 13(b) shows correspondingly the Fourier transform of the plasma density perturbations at the same time instants. In the lineouts as a function of $y$, the plasma flow is in three different regimes along the transverse direction: the flow is sub sonic for $y<1110 \lambda_{0}$, it is sonic around $\left(y \simeq 1110 \lambda_{0}\right)$, and super sonic for $y>1110 \lambda_{0}$.

In Fig. 13(a), during the initial stage of interaction $\left(2 k_{1} c_{s} t=\right.$ 70 , magenta curve) we only see small density perturbations in the three regions; however,the density perturbations in the respective regions increase with ongoing time with the development of a wave train close to the sonic region, along with a steeping in the density perturbation in the sonic region. In Fig. 13, the observed shift in the position of the shocklike structure around the sonic point $\left(v_{p} \equiv c_{s}\right.$, at $\left.\mathrm{y}=1110 \lambda_{0}\right)$ with time, can be attributed to the plasma flow. The corresponding Fourier analysis of the density perturbations in Fig. 13(b) shows that as the shock structure becomes more prominent with time, the spectra develop a plateau in the region $0.6 \lesssim k / k_{1} \leq 1$, a feature characteristic of shock generation. Also, as seen in Figs. 12(f),12(g) and 13(a), the typical size of the non-linear structures in the density perturbation corresponds to the size of laser speckles, and the perturbations are strongest in the vicinity where the plasma flow is sonic.

In the set of Figs. 14 we present and compare Fourier transforms of the nonlinear density perturbations for the three cases shown in Fig. 12(e)-12(g). In the same figures we also compare the wave number spectra with the change in damping coefficient $\hat{v}$ (accounting for the both collisional and Landau damping). Figure 14(a) shows the case with crossed-beam 


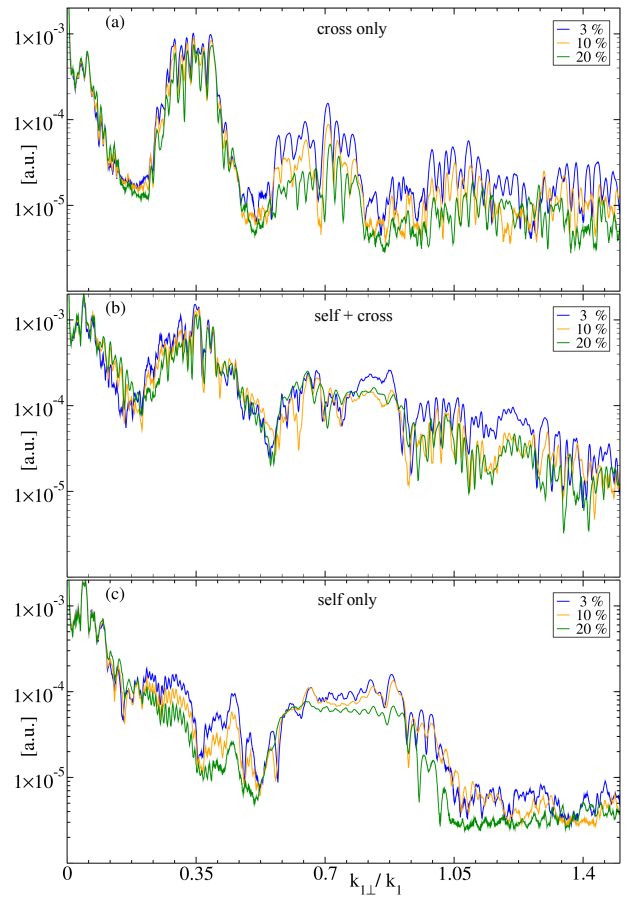

FIG. 14. (Color online) Spatial Fourier spectra of perturbed plasma density in the crossing regions, similar to the cases in figure 5(e)-(g) for different values of IAW damping, namely $\hat{v}=3 \%, 10 \%$ and $20 \%$ in blue, orange and green color, respectively. The presence of IAW harmonics is clearly visible in panel (a) for the SBS only case. The other spectra correspond to the cases (b) with both self-interaction and crossed-beam coupling and (c) self-interaction only. The value of $k_{1, y} / \mathrm{k}_{1}=0.35$ in the above spectra represents the normalized central CBET-SBS wave number.

coupling only. The spectrum peaks around the value expected for CBET-SBS at $k_{1, y} / k_{1}=2 \sin (\theta / 2) \sim 0.35$, followed along $k$ by other equally spaced peaks, corresponding to the second and third harmonics. This means that the CBET-SBS driven density perturbations can evolve into non-linear wave train structures when propagating through the plasma. These higher harmonics to the density perturbations associated with CBET-SBS decrease as a function of the IAW damping. In the spectrum corresponding to the case with self-interaction only, in Fig. 14(c), the contribution at the CBET-SBS wave number is evidently absent. More characteristic for this case is the plateau region in $k$ associated with the formation of isolated shock-like structures. The wave number spectra for the case with both self-interaction and crossed-beam coupling, in Fig. 14(b)), combines the features originating from both processes. For all the three cases we observe that the magnitude of the wave number components are reduced (note the log scale in Figs. 14) with the increase in the damping $\hat{v}$.

\section{v. CONCLUSION}

We have investigated the role of laser beam speckle structure in crossed beam energy transfer. It is an aspect of CBET that has been considered only in recent studies. We have demonstrated that the speckle structure plays an important role for laser beams crossing in a plasma with a flow when both self-focusing of intense laser speckles and forward SBS in RPP beams come into play. This can be expected for laser fluxes above $I_{L} \lambda_{0}^{2}>10^{14} \mathrm{~W} \mu \mathrm{m}^{2} / \mathrm{cm}^{2}$, i.e. for an intensity regime that is relevant for current ICF parameters.

For plasmas with inhomogeneous flow, where CBET occurs around sonic surfaces, the onset of self-focusing instability in speckles is enhanced, leading to a significant beam deflection and resulting in broadening of the angular light distribution in the transmitted laser beams. A regime with a maximum in the net transfer rate is attained for the intensity range of $1.5<I_{L} \lambda_{0}^{2} /\left(10^{14} \mathrm{~W} \mu m^{2} / \mathrm{cm}^{2}\right)<3$ with an observable deflection of the amplified beam to higher angles, and an increase in the angular width. Beam deflection and angular broadening may have significant impact on ICF laser energy deposition. Broadening of the angular distribution in both beams can be attributed to the plasma-induced smoothing and scattering off non linear IAW density perturbations.

The temporal incoherence due to plasma-induced smoothing observed in our simulations with RPP beams, see Fig. 10 (a), corresponds to correlation times of the order of $2 k_{1} c_{s} t_{\text {corr }} \sim 20$ being equivalent to a short $p s$ time scale for $\lambda_{0}=0.35 \mu \mathrm{m}, T_{e} \sim 3 \mathrm{keV}$, and angles $\theta \sim 20^{\circ}$. While we have not considered spatio-temporal smoothing, such as SSD, in our study, the result indicates that the effect of SSD with a bandwidth that is smaller than $50 \mathrm{GHz}$ should be quite marginal for the effects described in this study. Furthermore, it is known that the available SSD bandwidth on the National Ignition Facility has not prevented the onset of CBET. Higher SSD bandwidth may be available e.g. on the Omega laser facility at LLE Rochester, as this may be necessary for the direct drive ICF experiments.

Our study shows also that the presence of self-interaction results in shock-like structures with steepened wave fronts in the plasma density perturbation which can further lead to deflections of RPP beams in the inhomogeneous plasmas.

\section{ACKNOWLEDGMENTS}

This work has been carried out within the framework of the EUROfusion Consortium and has received funding from the Euratom research and training programme 2014-2018 and 2019-2020 under grant agreement No. 633053. The views and opinions expressed herein do not necessarily reflect those of the European Commission. We would like to thank the CPHT computer support team. WR was partially supported by the DOE Fusion Energy Sciences grant, user FWP100182.

${ }^{1}$ I. V. Igumenshchev, W. Seka, D. H. Edgell, D. T. Michel, D. H. Froula, V. N. Goncharov, R. S. Craxton, L. Divol, R. Epstein, R. Follett, J. H. Kelly, T. Z. Kosc, A. V. Maximov, R. L. McCrory, D. D. Meyerhofer, P. Michel, J. F. Myatt, T. C. Sangster, A. Shvydky, S. Skupsky, and C. Stoeckl, Phys. Plasmas 19, 056314(12) (2012).

${ }^{2}$ J. F. Myatt, J. Zhang, R. W. Short, A. V. Maximov, W. Seka, D. H. Froula, D. H. Edgell, D. T. Michel, I. V. Igumenshchev, D. E. Hinkel, P. Michel, and J. D. Moody, Phys. Plasmas 21, 055501 (2014). 
${ }^{3}$ J. F. Myatt, R. K. Follett, J. G. Shaw, D. H. Edgell, D. H. Froula, I. V. Igumenshchev, and V. N. Goncharov, Physics of Plasmas 24, 056308 (2017). ${ }^{4}$ R. K. Follett, D. H. Edgell, D. H. Froula, V. N. Goncharov, I. V. Igumenshchev, J. G. Shaw, and J. F. Myatt, Physics of Plasmas 24, 103128 (2017) ${ }^{5}$ D. H. Edgell, R. K. Follett, I. V. Igumenshchev, J. F. Myatt, J. G. Shaw, and D. H. Froula, Physics of Plasmas 24, 062706 (2017)

${ }^{6}$ P. Michel, L. Divol, E. A. Williams, C. A. Thomas, D. A. Callahan, S. Weber, S. W. Haan, J. D. Salmonson, N. B. Meezan, O. L. Landen, S. Dixit, D. E. Hinkel, M. J. Edwards, B. J. MacGowan, J. D. Lindl, S. H. Glenzer, and L. J. Suter, Phys. Plasmas 16, 042702 (2009).

${ }^{7}$ J. D. Moody, P. Michel, L. Divol, R. L. Berger, E. Bond, D. K. Bradley, D. A. Callahan, E. L. Dewald, S. Dixit, M. J. Edwards, S. Glenn, A. Hamza, C. Haynam, D. E. Hinkel, N. Izumi, O. Jones, J. D. Kilkenny, R. K. Kirkwood, J. L. Kline, W. L. Kruer, G. A. Kyrala, O. L. Landen, S. LePape, J. D. Lindl, B. J. MacGowan, N. B. Meezan, A. Nikroo, M. D. Rosen, M. B. Schneider, D. J. Strozzi, L. J. Suter, C. A. Thomas, R. P. J. Town, K. Widmann, E. A. Williams, L. J. Atherton, S. H. Glenzer, and E. I. Moses, Nat. Phys. , 344 (2012).

${ }^{8}$ P. Michel, L. Divol, E. A. Williams, S. Weber, C. A. Thomas, D. A. Callahan, S. W. Haan, J. D. Salmonson, S. Dixit, D. E. Hinkel, M. J. Edwards, B. J. MacGowan, J. D. Lindl, S. H. Glenzer, and L. J. Suter, Phys. Rev. Lett. 102, 025004 (2009).

${ }^{9}$ L. Lancia, J.-R. Marquès, M. Nakatsutsumi, C. Riconda, S. Weber S. Hüller, A. Mančić, P. Antici, V. T. Tikhonchuk, A. Héron, P. Audebert, and J. Fuchs, Phys. Rev. Lett. 104, 025001 (2010)

${ }^{10}$ E. Guillaume, K. Humphrey, H. Nakamura, R. M. G. M. Trines, R. Heathcote, M. Galimberti, Y. Amano, D. Doria, G. Hicks, E. Higson, S. Kar, G. Sarri, M. Skramic, J. Swain, K. Tang, J. Weston, P. Zak, E. P. Alves, R. A. Fonseca, F. Fiúza, H. Habara, K. A. Tanaka, R. Bingham, M. Borghesi, Z. Najmudin, L. O. Silva, and P. A. Norreys, High Power Laser Sci. Eng. 2, e33 (2014)

${ }^{11}$ D. Turnbull, C. Goyon, G. E. Kemp, B. B. Pollock, D. Mariscal, L. Divol, J. S. Ross, S. Patankar, J. D. Moody, and P. Michel, Phys. Rev. Lett. 118 015001 (2017)

${ }^{12}$ J. D. Ludwig, P. Michel, T. Chapman, M. A. Belyaev, and W. Rozmus, Physics of Plasmas 26, 113108 (2019)

${ }^{13}$ W. L. Kruer, S. C. Wilks, B. B. Afeyan, and R. K. Kirkwood, Phys. Plasma 3, 382 (1996).

${ }^{14}$ R. K. Kirkwood, B. B. Afeyan, W. L. Kruer, B. J. MacGowan, J. D. Moody, D. S. Montgomery, D. M. Pennington, T. L. Weiland, and S. C. Wilks, Phys. Rev. Lett. 76, 2065 (1996).

${ }^{15}$ V. V. Eliseev, W. Rozmus, V. T. Tikhonchuk, and C. E. Capjack, Physics of Plasmas 3, 2215 (1996)

${ }^{16}$ G. Raj and S. Hüller, Phys. Rev. Lett. 118, 055002 (2017).

${ }^{17}$ C. Labaune, H. A. Baldis, E. Schifano, B. S. Bauer, A. Maximov, I. Ourdev, W. Rozmus, and D. Pesme, Phys. Rev. Lett. 85, 1658 (2000).

${ }^{18}$ C. Labaune, H. A. Baldis, B. Cohen, W. Rozmus, S. Depierreux, E. Schifano, B. S. Bauer, and A. Michard, Phys. Plasmas 6, 2048 (1999)

${ }^{19}$ A. Colaïtis, S. Hüller, D. Pesme, G. Duchateau, and V. T. Tikhonchuk, Phys. Plasmas 23, 032118 (2016).

${ }^{20}$ Y. Kato and K. Mima, Appl. Phys. B 29, 186 (1982).

${ }^{21}$ H. A. Rose and D. F. DuBois, Phys. Fluids B 5, 3337 (1993)

${ }^{22}$ H. A. Rose and D. F. DuBois, Phys. Fluids B 5, 590 (1993).

${ }^{23}$ H. A. Rose and S. Ghosal, Phys. Plasmas 5, 1461 (1998).

${ }^{24}$ H. A. Rose and S. Ghosal, Phys. Plasmas 5, 775 (1998).

${ }^{25}$ J. A. F. Hittinger, M. R. Dorr, R. L. Berger, and E. A. Williams, J. Comput. Phys. 209, 695 (2005)

${ }^{26}$ P. Michel, S. H. Glenzer, L. Divol, D. K. Bradley, D. Callahan, S. Dixit, S. Glenn, D. Hinkel, R. K. Kirkwood, J. L. Kline, W. L. Kruer, G. A. Kyrala, S. Le Pape, N. B. Meezan, R. Town, K. Widmann, E. A. Williams, B. J. MacGowan, J. Lindl, and L. J. Suter, Phys. Plasmas 17, 056305 (2010).

${ }^{27}$ L. Yin, B. J. Albright, D. J. Stark, W. D. Nystrom, R. F. Bird, and K. J. Bowers, Physics of Plasmas 26, 082708 (2019).

${ }^{28}$ A. Colaitis, S. Hüller, V. T. Tikhonchuk, D. Pesme, G. Duchateau, and A. Porzio, J. Phys.: Conf. Ser. 717, 012096 (2016)

${ }^{29} \mathrm{C}$. J. Randall, J. R. Albritton, and J. J. Thomson, Physics of Fluids 24, 1474 (1981)

${ }^{30}$ S. Hüller, P. Mulser, and A. M. Rubenchik, Physics of Fluids B 3, 3339 (1991)

${ }^{31}$ R. W. Short, R. Bingham, and E. A. Williams, Phys. Fluids 25, 2302
(1982).

${ }^{32}$ A. J. Schmitt, Physics of Fluids B 1, 1287 (1989).

${ }^{33}$ H. A. Rose, Phys. Plasmas 3, 1709 (1996).

${ }^{34}$ D. E. Hinkel, E. A. Williams, and C. H. Still, Phys. Rev. Lett. 77, 1298 (1996).

${ }^{35}$ B. Bezzerides, Physics of Plasmas 5, 2712 (1998)

${ }^{36}$ J. Candy, W. Rozmus, and V. T. Tikhonchuk, Phys. Rev. Lett. 65, 1889 (1990).

${ }^{37}$ S. Hüller, Physics of Fluids B 3, 3317 (1991).

${ }^{38} \mathrm{~V}$. I. Karpman, Non-linear waves in dispersive media (Pergamon Press Oxford, New York, 1974).

${ }^{39}$ D. E. Hinkel, M. B. Schneider, E. A. Williams, A. B. Langdon, L. J. Suter, and P. T. Springer in in Inertial Fusion Sciences and Applications 2003 , edited by J. M.-t.-V. B. A. Hammel, D. D. Meyerhofer and H. Azechi (Elsevier, Amsterdam, 2004) pp. 242-246.

${ }^{40}$ E. A. Williams, D. E. Hinkel, and H. J. A., in in Inertial Fusion Sciences and Applications 2003, edited by J. M.-t.-V. B. A. Hammel, D. D. Meyerhofer and H. Azechi (Elsevier, Amsterdam, 2004) pp. 252-255.

${ }^{41}$ D. E. Hinkel, D. A. Callahan, A. B. Langdon, S. H. Langer, C. H. Still, and E. A. Williams, Phys. Plasmas 15, 056314 (2008)

${ }^{42}$ R. K. Kirkwood, J. D. Moody, A. B. Langdon, B. I. Cohen, E. A. Williams, M. R. Dorr, J. A. Hittinger, R. Berger, P. E. Young, L. J. Suter, L. Divol, S. H. Glenzer, O. L. Landen, and W. Seka, Phys. Rev. Lett. 89, 215003 (2002)

${ }^{43}$ R. K. Kirkwood, E. A. Williams, B. I. Cohen, L. Divol, M. R. Dorr, J. A. Hittinger, A. B. Langdon, C. Niemann, J. Moody, L. J. Suter, and O. L. Landen, Physics of Plasmas 12, 112701 (2005).

${ }^{44}$ R. K. Kirkwood, P. Michel, R. London, J. D. Moody, E. Dewald, L. Yin, J. Kline, D. Hinkel, D. Callahan, N. Meezan, E. Williams, L. Divol, B. L. Albright, K. J. Bowers, E. Bond, H. Rose, Y. Ping, T. L. Wang, C. Joshi, W. Seka, N. J. Fisch, D. Turnbull, S. Suckewer, J. S. Wurtele, S. Glenzer, L. Suter, C. Haynam, O. Landen, and B. J. Macgowan, Phys. Plasmas 18 056311 (2011)

${ }^{45}$ C. Neuville, C. Baccou, A. Debayle, P.-E. Masson-Laborde, S. Hüller, M. Casanova, D. Marion, P. Loiseau, K. Glize, C. Labaune, and S. Depierreux, Phys. Rev. Lett. 117, 145001 (2016).

${ }^{46}$ C. Neuville, K. Glize, P.-E. Masson-Laborde, P. Loiseau, S. Hüller, A. Debayle, C. Baccou, M. Casanova, C. Labaune, and S. Depierreux, Physics of Plasmas 24, 112110 (2017)

${ }^{47}$ R. K. Kirkwood, B. J. MacGowan, D. S. Montgomery, B. B. Afeyan, W. L. Kruer, D. M. Pennington, S. C. Wilks, J. D. Moody, K. Wharton, C. A. Back, K. G. Estabrook, S. H. Glenzer, M. A. Blain, R. L. Berger, D. E. Hinkel, B. F. Lasinski, E. A. Williams, D. Munro, B. H. Wilde, and C. Rousseaux, Phys. Plasmas 4, 1800 (1997).

${ }^{48}$ D. S. Montgomery, B. B. Afeyan, J. A. Cobble, J. C. Fernández, M. D. Wilke, S. H. Glenzer, R. K. Kirkwood, B. J. MacGowan, J. D. Moody, E. L. Lindman, D. H. Munro, B. H. Wilde, H. A. Rose, D. F. Dubois, B. Bezzerides, and H. X. Vu, Phys. Plasmas 5, 1973 (1998).

${ }^{49}$ K. B. Wharton, R. K. Kirkwood, S. H. Glenzer, K. G. Estabrook, B. B. Afeyan, B. I. Cohen, J. D. Moody, B. J. MacGowan, and C. Joshi, Phys. Plasmas 6, 2144 (1999).

${ }^{50} \mathrm{M}$. Casanova, (2009), private communication

${ }^{51}$ D. Pesme, S. Hüller, J. Myatt, C. Riconda, A. Maximov, V. T. Tikhonchuk, C. Labaune, J. Fuchs, S. Depierreux, and H. A. Baldis, Plasma Phys. Controlled Fusion 44, B53 (2002).

${ }^{52}$ S. Hüller, P. E. Masson-Laborde, D. Pesme, M. Casanova, F. Detering, and A. Maximov, Phys. Plasmas 13, 022703 (2006)

${ }^{53}$ C. J. McKinstrie, J. S. Li, R. E. Giacone, and H. X. Vu, Phys. Plasmas 3 , 2686 (1996).

${ }^{54}$ A. V. Maximov, I. G. Ourdev, D. Pesme, W. Rozmus, V. T. Tikhonchuk, and C. E. Capjack, Phys. Plasmas 8, 1319 (2001).

${ }^{55}$ A. Colaitis, G. Duchateau, X. Ribeyre, and V. Tikhonchuk, Phys. Rev. E 91, 013102 (2015)

M. N. Rosenbluth, Phys. Rev. Lett. 29, 565 (1972).

${ }^{57}$ M. R. Amin, C. E. Capjack, P. Frycz, W. Rozmus, and V. T. Tikhonchuk, Physics of Fluids B 5, 3748 (1993).

${ }^{58}$ A. J. Schmitt and B. B. Afeyan, Phys. Plasmas 5, 503 (1998).

${ }^{59}$ D. S. Montgomery, R. P. Johnson, H. A. Rose, J. A. Cobble, and J. C. Fernández, Phys. Rev. Lett. 84, 678 (2000). 